\title{
Seismic rays in media with slight lateral variation in velocity
}

\author{
Beverley J. Moore \\ School of Mathematical Sciences, University of Technology, Sydney, PO Box 123, Broadway, NSW 2007, Australia
}

Accepted 1990 October 29. Received 1990 October 29; in original form 1990 April 3

\begin{abstract}
SUMMARY
In this paper, the method of small perturbations is applied to the ray and energy transport equations in an investigation of the effect on the propagation of seismic rays through a layer of fixed thickness of having weak lateral inhomogeneities superimposed on an unperturbed velocity field which varies with depth only. Both plane and spherical geometries are studied. This more fundamental approach highlights inadequacies of the more commonly used Hamiltonian method. In the course of calculations, simple expressions are also obtained for the various components of the propagator matrices for rays in vertically and radially heterogeneous media; these are useful in the study of Gaussian beams and paraxial ray theory in such media and require the calculation of just two integrals along the unperturbed ray path. In particular, these integrals are evaluated analytically for the special case of weak lateral velocity inhomogeneities superimposed on a constant gradient of quadratic slowness. It is found that the perturbation to ray geometry may include a component directed along the ray at the point of observation without violating Fermat's principle and that this may be neglected only when slowness does not vary greatly along the unperturbed ray path. Such perturbations are usually ignored in paraxial ray theory. Expressions are also obtained for the first-order corrections to traveltime, amplitude, surface slowness and polarization in such media; the approach used in deriving these expressions gives insight into the various complicating factors which need to be considered in inverse modelling problems involving such media.
\end{abstract}

Key words: seismic ray theory, slight lateral variations, ray perturbation theory.

\section{INTRODUCTION}

In recent years, there has been an upsurge of interest in the study of seismic wave propagation in laterally varying earth models. This has been prompted by attempts to map heterogeneous structures within the Earth in greater and greater detail. Even with all the progress made in developing techniques for extracting structural information about the uppermost few kilometres of the Earth from reflection data obtained in the search for hydrocarbons, the need still remains for more refined methods of estimating the lateral velocity inhomogeneity and accounting for it accurately in the migration process.

Most investigations of lateral variations within the Earth have used some form of perturbation to ray theory. For example, Aki, Christoffersson \& Husebye (1977) developed a flexible block modelling procedure for 3-D mappings of seismic velocity structure using the observed traveltime residuals. Their method involves the determination of the velocity (or slowness) perturbations in individual cells; perturbations in the actual ray paths due to fluctuations in the seismic velocity field are neglected on the basis of Fermat's Principle. Nolet (1987) cites many applications of this technique both in the study of large continental areas and also in the study of very local structures.

However, there is much more information in the seismic record than just a list of traveltimes and currently there is considerable interest in using other forms of data from the seismic record to assist in the determination of subsurface structure. For example, Sutton \& Moore (1987) work with reflection data and use both two-way traveltimes and stacking velocities from an unmigrated stacked section in their determination of subsurface structure; they allow lateral variations in the velocity model but handle the curved rays by means of straight rays with corrections determined from the results presented in Moore (1980) which are applicable to this problem where fluctuations in the interval velocities across the layer are not too large. Of course, the possibility of replacing curved rays by straight rays with corrections becomes particularly attractive in 
problems involving layers of variable thickness as the layer boundaries are themselves curved and Moore (1989) outlines the principles of such a ray tracing procedure and its advantages over using straight rays without corrections.

Nevertheless, the real focus of recent developments has been the search for a more adequate way to describe amplitude fluctuations in the high-frequency limit-one that has no singularities at caustics, etc. This has given rise to the concept of Gaussian beams (Červený, Popov \& Pšencík 1982; Cervený \& Pšenčík 1984) and the application of Maslov theory (Chapman \& Drummond 1982). Madariaga (1984) has shown that Gaussian beam summation is an analytic continuation to complex values of position and slowness of the WKB method proposed by Chapman (1978); Chapman (1985) points out that the WKBJ seismogram is the 1-D version of the Maslov seismogram and that the Maslov seismogram is a special limiting case of the Gaussian beam method corresponding to infinite beam width.

All these generalizations of the ray method are formulated in terms of the solutions to the dynamic ray tracing equations in association with the kinematic ray tracing equations. Cervený (1985) has noted that whereas the ray method needs only real-valued solutions of the dynamic ray tracing equations, Gaussian beams need complex-valued solutions of these equations, but that these may be expressed as linear combinations of real-valued solutions obtained by the paraxial ray approximation. Farra \& Madariaga (1987) go on to observe that not only is much of this work on Gaussian beams based on the paraxial approximation, but that this approximation is actually derived from first-order ray perturbation theory and they have attempted a unified approach to the study of both paraxial rays and perturbed rays in slightly heterogeneous media using the propagator formalism. Indeed, the results they obtain for the perturbed rays in a medium with a homogeneous reference model are in agreement with the results obtained by Moore (1980, to be referred to hereafter as paper I) using quite a different formalism based on the approach of Keller (1962) who applied the method of small perturbations to the ray and energy transport equations. However, paraxial ray theory is limited to the study of the transverse components of the deviation in ray geometry and does not fully describe the geometry of perturbed rays in a medium whose reference model is not homogenous. It will be shown in this paper that in such media, the perturbation to ray geometry may include a component directed along the ray at the point of observation without violating Fermat's principle and that this may be neglected only when slowness does not vary greatly along the unperturbed ray path. Furthermore, this tangential perturbation to ray geometry can affect both the amplitude and polarization fluctuations.

The method of small perturbations is applied to the ray and energy transport equations for material in which small lateral variations are superimposed on any kind of depth variation of velocity; results are obtained in terms of the ray paths and amplitudes of the unperturbed rays as well as the structure of the heterogeneity. This more fundamental approach will highlight inadequacies of the more commonly used Hamiltonian method. Both plane and spherical geometries are studied and it is assumed that the trajectories of the rays in the unperturbed reference medium are known.
The effect on ray geometry of weak lateral velocity inhomogeneities superimposed on a constant gradient in quadratic slowness is considered in Section 3 of this paper. Expressions are also obtained for the various components of the propagator matrices for rays in vertically and radially heterogeneous media; these require the calculation of just two integrals along the unperturbed ray path rather than the usual numerical solution of a system of ordinary differential equations.

\section{PERTURBATION TO SEISMIC RAY GEOMETRY RESULTING FROM SLIGHT LATERAL VARIATIONS IN VELOCITY}

A ray $\mathbf{x}(s)$ propagating through an isotopic elastic medium whose velocity field is given by $\alpha(x)=u^{-1}(x)$ satisfies the equation

$\frac{d}{d s}\left(u \frac{d \mathbf{x}}{d s}\right)=\nabla u$,

where $s$ denotes arclength along the ray from some reference point, and $\mathbf{x}$ denotes the position vector of a point on the ray relative to the origin of the coordinate system.

It is convenient to express the equation more succinctly as

$g(\mathbf{x}, \dot{\mathbf{x}}, \ddot{\mathbf{x}})=0$

where

$g(\mathbf{x}, \dot{\mathbf{x}}, \ddot{\mathbf{x}})=\frac{d}{d s}\left(u(\mathbf{x}) \frac{d \mathbf{x}}{d s}\right)-\nabla u(\mathbf{x})$

and denotes differentiation with respect to arclength along the ray.

We now consider the propagation of seismic rays through a medium those velocity field deviates only slightly from the depth-varying field $\alpha_{0}\left(x_{3}\right)=u_{0}^{-1}\left(x_{3}\right)$ where $x_{3}$ denotes either the vertical coordinate of position in plane geometry or $|x|$ in spherical geometry. In this case, we represent the slowness field by

$u(\mathbf{x})=u_{0}\left(x_{3}\right)\left[1+\varepsilon f_{1}(\mathbf{x})\right], \quad\left|f_{1}\right|<1$,

where $\varepsilon$ is a small parameter measuring the deviation of the medium from its unperturbed state.

The standard approach (see Farra \& Madariaga 1987) has been to use Hamilton's method. However, although a simple form of the Hamiltonian,

$H=\frac{1}{2}\left(\mathbf{p} \cdot \mathbf{p}-u^{2}\right)$

is obtained when the parameter along the ray is

$w=w_{0}+\int_{0}^{s} \frac{d s^{\prime \prime}}{u\left(s^{\prime \prime}\right)}$

this parametrization of the ray is not appropriate for use in a perturbation analysis problem because the scale length of this parameter varies with position. On the other hand, it is not easy to find an expression for the Hamiltonian when arclength is the parameter along the ray. Therefore, we take a more fundamental approach.

The ray $\mathbf{x}(s ; \varepsilon)$ in such a medium is found $(c f$. paper $\mathrm{I})$ by determining its derivatives with respect to $\varepsilon$ at $\varepsilon=0$ and 
then representing $\mathbf{x}$ by means of its Taylor series in $\varepsilon$ :

$\mathbf{x}(s ; \varepsilon)=\mathbf{x}^{0}(s)+\varepsilon \mathbf{x}^{\varepsilon}(s)+0\left(\varepsilon^{2}\right)$.

The geometry of both unperturbed ray and first-order correction to the ray path are illustrated in Fig. 1.

Setting $\varepsilon=0$ in equations (2) and (4) gives the usual equations for the ray in the unperturbed medium, subject to appropriate initial and boundary conditions:

$g_{0}\left(\mathbf{x}^{0}, \dot{\mathbf{x}}^{0}, \ddot{\mathbf{x}}^{0}\right)=\frac{d}{d s}\left(u_{0}\left(x_{3}^{0}\right) \frac{d \mathbf{x}^{0}}{d s}\right)-\nabla u_{0}\left(x_{3}^{0}\right)=\mathbf{0}$.

Thus, the zeroth-order solution $\mathbf{x}^{0}(s)$ has the same geometry as the ray leaving the same reference point in the same direction in the unperturbed depth-varying medium. In plane geometry, the first two equations of (7) are readily integrated to give

$u_{0}\left(x_{3}^{0}\right) \frac{d x_{i}^{0}}{d s}=p_{i}, \quad$ constants, $\quad i=1,2$.

Without loss of generality, it is possible to set $p_{2}=0$; that is, to choose a particular orientation of the Cartesian coordinate axes so that the zeroth-order solution is confined to the plane $x_{2}=0$. Writing $p$ for $p_{1}$, we obtain the following expression for the vector tangent to the ray from equation (8):

$$
\begin{aligned}
\frac{d \mathbf{x}^{0}}{d s} & =\left[p \alpha_{0}\left(x_{3}^{0}\right), 0, \sqrt{1-p^{2} \alpha_{0}^{2}\left(x_{3}^{0}\right)}\right] \\
& =[\sin \theta(s), 0, \cos \theta(s)],
\end{aligned}
$$

where $\theta$ denotes the angle between the direction of the ray and the vertical axis.

Generalizing the notation of equation (8) we may define the vector

$\mathbf{p}^{0}=\mathrm{u}_{0} \frac{d \mathbf{x}^{0}}{d s}=\left[p, 0, \sqrt{u_{0}^{2}\left(x_{3}^{0}\right)-p^{2}}\right]$.

Equation (9) is, in fact a statement of Snell's law in the vertically stratified medium. Furthermore from the Frenet formulae and equation (7) we see that $\mathbf{x}^{0}(s)$ is a torsionless ray of curvature $\left|\nabla^{T} \ln u_{0}\left(x_{3}^{0}\right)\right|$ where the transverse gradient operator is defined to be

$$
\nabla^{T}=\nabla-\frac{d \mathbf{x}^{0}}{d s}\left(\frac{d \mathbf{x}^{0}}{d s} \cdot \nabla\right)
$$

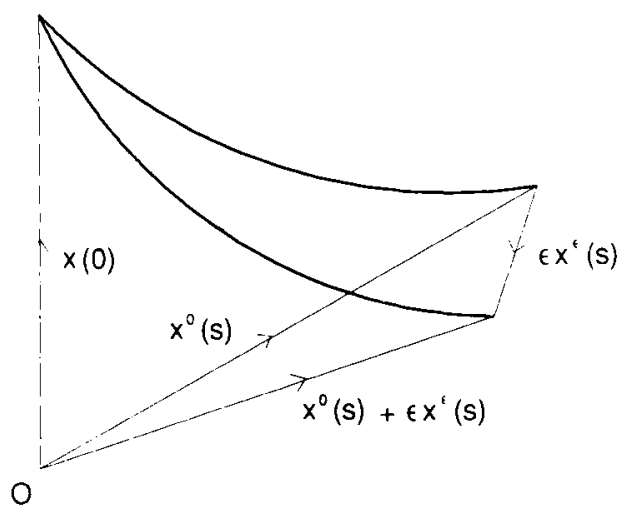

Figure 1. Diagram showing first-order correction to the unperturbed ray path. The position vectors of typical points on these rays are indicated.
That is, the curvature of the ray $x^{0}(s)$ is governed by the variation in $u_{0}$ across the wavefront of the disturbance in the neighbourhood of each point on the ray path.

In spherical geometry, we take the vector product of equation (7) with $x^{0}$ and integrate to obtain

$u_{0} x^{0} \times \frac{d \mathbf{x}^{0}}{d s}=\mathbf{p}, \quad$ a constant vector,

that is, the unperturbed ray is again confined to a single plane. This fact may be used in choosing the orientation of the spherical polar coordinates $(r, \theta, \phi)$ so that the plane of the unperturbed ray is simply $\phi=\phi_{0}$, a constant. Indeed, without loss of generality we may take $\phi_{0}=0$. The properties of this coordinate system are outlined in Appendix B. The normal to the plane of the unperturbed ray is given by $\hat{\phi}$, a constant vector. Now,

$\mathbf{p}=p \hat{\boldsymbol{\phi}}=u_{0} \mathbf{y}_{0} \times \mathbf{e}=u_{0}\left|\mathbf{y}_{0}\right| \sin \psi_{0} \hat{\boldsymbol{\phi}}$

where

$\mathrm{y}_{0}=\mathrm{x}^{\mathrm{0}}(0), \quad \mathrm{e}=\frac{d \mathrm{x}^{\mathrm{o}}}{d s}(0)$

and $\psi_{0}$ is the angle between $\mathbf{y}_{0}$ and $\mathbf{e}$.

Thus

$\mathbf{e}=\sin \psi_{0} \hat{\boldsymbol{\theta}}(0)+\cos \psi_{0} \hat{\mathbf{r}}(0)$

and more generally

$\begin{aligned} \frac{d \mathbf{x}^{0}}{d s} & =\sin \psi(s) \hat{\boldsymbol{\theta}}(s)+\cos \psi(s) \hat{\mathbf{r}}(s) \\ & =\frac{p}{r_{0} u_{0}\left(r_{0}\right)} \hat{\boldsymbol{\theta}}(s)+\sqrt{1-\left(\frac{p}{r_{0} u_{0}\left(r_{0}\right)}\right)^{2}} \hat{\mathbf{r}}(s)\end{aligned}$

where $r_{0}=\left|\mathbf{x}^{0}\right|$.

Equation (14) is a statement of Snell's law in the spherically symmetric medium. Furthermore, writing $\mathbf{x}^{0}(s)=r_{0}(s) \hat{\mathbf{r}}(s)$ and using equation (B-4) gives an alternative representation of $d \mathbf{x}^{0} / d s$ :

$\frac{d \mathbf{x}^{0}}{d s}=r_{0} \dot{\theta} \hat{\boldsymbol{\theta}}(s)+\dot{r}_{0} \hat{r}(s)$

where denotes differentiation with respect to arclength along the ray.

Thus we are able to obtain expressions for $\dot{\theta}$ and $\dot{r}_{0}$ on the unperturbed ray.

We now rewrite equation (2) in the form

$g(\mathbf{x}, \dot{\mathbf{x}}, \ddot{\mathbf{x}})=g_{0}(\mathbf{x}, \dot{\mathbf{x}}, \ddot{\mathbf{x}})+\varepsilon g_{1}(\mathbf{x}, \dot{\mathbf{x}}, \ddot{\mathbf{x}})+0\left(\varepsilon^{2}\right)=0$

where $\mathrm{x}$ is given by equation (6), $g_{0}$ is given by equation (3) with $u(x)$ replaced by $u_{0}\left(x_{3}\right)$, and $g_{1}$ is given by equation (3) with $u(\mathbf{x})$ replaced by $f_{1}(\mathbf{x}) u_{0}\left(x_{3}\right)$.

Differentiating equation (2) with respect to $\varepsilon$ and setting $\varepsilon=0$ gives

$\left(\mathbf{x}^{\varepsilon} \cdot \nabla_{\mathbf{x}} g_{0}+\dot{\mathbf{x}}^{\varepsilon} \cdot \nabla_{\dot{\mathbf{x}} g_{0}}+\ddot{\mathbf{x}}^{\varepsilon} \cdot \nabla_{\ddot{2} g_{0}}+g_{1}\right)_{\substack{\mathbf{x}=\mathbf{x}^{0} \\ \dot{\mathbf{x}}=\dot{\mathbf{x}}^{0} \\ \overrightarrow{\mathbf{x}}=\mathbf{x}^{0}}}=\mathbf{0}$ 
that is,

$$
\begin{aligned}
& \frac{d}{d s}\left(u_{0} \frac{d \mathrm{x}^{\varepsilon}}{d s}\right)+\frac{d}{d s}\left(\mathrm{x}^{\varepsilon} \cdot \nabla u_{0} \frac{d \mathbf{x}^{0}}{d s}\right)-\mathrm{x}^{\varepsilon} \cdot \nabla\left(\nabla u_{0}\right) \\
& \quad=u_{0} \nabla^{T} f_{1}\left(\mathrm{x}^{\mathrm{o}}\right) .
\end{aligned}
$$

Of course, it is possible to simplify equation (18) utilizing the fact that the unperturbed velocity field varies only with depth $x_{3}$ and contains no lateral variations.

Since we are studying the geometry of the ray which passes through the reference point $x^{0}(0)$ in a particular direction $d x^{0} / d s(0)$, the appropriate initial conditions to use with equation (18) are

$\mathrm{x}^{\varepsilon}(0)=0 \quad$ and $\quad \frac{d \mathrm{x}^{\varepsilon}}{d s}(0)=\mathbf{0}$.

However, in some applications, it might be desirable to perform the calculations of $x^{\varepsilon}$ in several stages. Therefore, equation (18) is solved in Appendices $A$ and $C$ subject to non-zero initial conditions. This equation is also, in essence, the equation which needs to be solved in order to determine the 'propagators' for the unperturbed ray. These 'propagators' describe how small variations in the initial position and direction of the ray are propagated along the ray and thus they contain information on the variation in the ray field across the wavefront in the vertically varying velocity field in the vicinity of the particular ray of interest. Equation (18) with $f_{1} \equiv 0$ effectively becomes the equation for paraxial rays in for the unperturbed medium. In this context, $\varepsilon$ should be interpreted as a small parameter measuring the variation in the initial conditions rather than its usual meaning of measuring the deviation of the medium from its unperturbed state. Indeed, equation (18) with $f_{1} \equiv 0$ can be expressed in a form similar to that proposed by Farra \& Madariaga (1987) for the numerical calculation of the paraxial rays, viz.

$\delta \dot{\mathbf{y}}=\left(\begin{array}{c}\delta \dot{\mathbf{q}} \\ \delta \dot{\mathbf{p}}\end{array}\right)=\mathbf{A}(s)\left(\begin{array}{c}\delta \mathbf{q} \\ \delta \mathbf{p}\end{array}\right)$

where

$\mathbf{A}=\left(\begin{array}{cc}-\mathbf{p} \frac{\nabla u_{0}}{u_{0}^{2}} & \frac{1}{u_{0}} \mathbf{I} \\ \nabla \nabla u_{0} & \mathbf{0}\end{array}\right)$

Here $q$ and $p$ refer to the full 3-D displacements and slownesses so that, in general $\mathbf{p} \neq \mathbf{0}$. Of course, in ray centred coordinates, the matrix $\mathbf{A}$ relating transverse components of $\mathbf{q}$ and $\mathbf{p}$ is the same as that obtained by Farra \& Madariaga (1987).

If we let $\varepsilon \mathbf{q}(s)$ denote the first-order correction to position on the unperturbed ray and $\varepsilon \delta(s)$ denote the first-order correction to direction on the unperturbed ray both resulting from the small changes in the initial conditions, and introduce a coordinate $n$ measuring distance perpendicular to the unperturbed ray in the 1-3 plane (for plane geometry) or the plane $\phi_{0}=0$ in spherical geometry then it follows from the solution in Appendices $A$ and $C$ that if $q(0)$ has no component along the ray, then the transverse components of $q(s)$ and $\delta(s)$ (with the $n$-component listed before the 2-component) are given by

$$
\left(\begin{array}{c}
\mathbf{q}^{T}(s) \\
\delta(s)
\end{array}\right)=\Pi(s, 0)\left(\begin{array}{c}
\mathbf{q}^{T}(0) \\
\delta(0)
\end{array}\right)
$$

where

$\Pi(s, 0)=\left(\begin{array}{ll}\mathbf{P}_{1}(s, 0) & \mathbf{P}_{2}(s, 0) \\ \mathbf{P}_{3}(s, 0) & \mathbf{P}_{4}(s, 0)\end{array}\right)$

and the submatrices $\boldsymbol{P}_{k}$ are diagonal matrices whose elements depend only on the unperturbed velocity field and the associated geometry of the unperturbed ray as outlined below. Only two ray integrals need to be evaluated to specify $\Pi$ completely:

$I_{1}=\int_{0}^{s} \frac{p_{3}^{0}(0) p_{3}^{0}(s) u_{0}\left(s^{\prime \prime}\right)}{\left[p_{3}^{0}\left(s^{\prime \prime}\right)\right]^{2}} d s^{\prime \prime}$,

and

$I_{2}=\int_{0}^{s} \frac{p_{3}^{0}(0) p_{3}^{0}(s)}{\left[p_{3}^{0}\left(s^{\prime \prime}\right)\right]^{2}} d s^{\prime \prime}$,

where

$p_{3}^{0}=\left\{\begin{array}{lr}\sqrt{u_{2}^{0}-p^{2}}, & \text { for plane geometry, } \\ \sqrt{\left(r_{0} u_{0}\right)^{2}-p^{2}}, & \text { for spherical geometry. }\end{array}\right.$

Although these integrals appear to have singularities at the turning points of the unperturbed rays, the combination of terms in $\Pi(s, 0)$ is such that all elements of $\Pi$ remain finite at any turning point. (This is illustrated in Section 3 for the case of weak lateral velocity inhomogeneities superimposed on constant gradient of quadratic slowness.)

Now, the curvature of the unperturbed ray at $x^{0}(s)$ is given by

$\kappa(s)=\left\|\frac{\hat{\mathbf{n}}}{d s}\right\|=\left\|\frac{d^{2} \mathbf{x}^{0}}{d s^{2}}\right\|= \begin{cases}\frac{p u_{0}^{\prime}(s)}{u_{0}^{2}(s)}, & \text { for plane geometry, } \\ \frac{p \frac{d u_{0}}{d r_{0}}(s)}{r_{0}(s) u_{0}^{2}(s)}, & \text { for spherical geometry. }\end{cases}$

Because $p_{3}^{0}$ is $r_{0}$ times the radial component of the slowness vector in spherical geometry, it is convenient to define the related quantity:

$$
L(0)=\frac{1}{p}\left(\frac{d x_{1}^{0}}{d s}\right)^{2} \frac{d p_{3}^{0}}{d s}=\left\{\begin{array}{l}
\kappa(0), \quad \text { for plane geometry, } \\
\frac{p \frac{d}{d r_{0}}\left(r_{0} u_{0}\right)}{\left(r_{0} u_{0}\right)^{2}} \\
\text { for spherical geometry. }
\end{array} \mid \begin{array}{l}
s=0 \\
\text { for }
\end{array}\right.
$$

Then, the submatrices in equation (21) are given by the following.

$\mathbf{P}_{1}(s, 0)=\operatorname{diag}\left[R_{1}(s, 0), R_{2}(s, 0)\right]$,

where

$$
\begin{aligned}
& R_{1}(s, 0)=\frac{p_{3}^{0}(s) u_{0}(0)-p L(0) I_{1}}{p_{3}^{0}(0) u_{0}(s)} \\
& R_{2}(s, 0)=\left\{\begin{array}{l}
1, \quad \text { for plane geometry, } \\
\frac{u_{0}(0) r_{0}(s)}{p} \hat{\theta}(s) \cdot \mathbf{e}, \quad \text { for spherical geometry }
\end{array}\right. \\
& \mathbf{P}_{2}(s, 0)=\operatorname{diag}\left[S_{1}(s, 0), S_{2}(s, 0)\right]
\end{aligned}
$$


where

$$
\begin{aligned}
& S_{1}(s, 0)=\frac{I_{1}}{u_{0}(s)}, \\
& S_{2}(s, 0)=\left\{\begin{array}{l}
\int_{0}^{s} \frac{u_{0}(0)}{u_{0}\left(s^{\prime \prime}\right)} d s^{\prime \prime}=\frac{u_{0}(0)}{p}\left[x_{1}^{0}(s)-x_{1}^{0}(0)\right], \\
\quad \text { for plane geometry, } \\
-\frac{u_{0}(0) r_{0}(0)}{p} r_{0}(s) \hat{\boldsymbol{\theta}}(s) \cdot \hat{\mathbf{r}}(0), \\
\text { for spherical geometry. }
\end{array}\right. \\
& \mathbf{P}_{3}(s, 0)=\operatorname{diag}\left[R_{4}(s, 0), R_{5}(s, 0)\right],
\end{aligned}
$$

$R_{4}(s, 0)=\frac{p}{p_{3}^{0}(0)}\left[\kappa(s)-L(0) S_{4}(s, 0)\right]+V$,

$V= \begin{cases}0, & \text { for plane geometry, } \\ \frac{u_{0}(0)}{p_{3}^{0}(0)}, & \text { for spherical geometry, }\end{cases}$

$R_{5}(s, 0)=\left\{\begin{array}{l}0, \quad \text { for plane geometry, } \\ \frac{u_{0}(0)}{p} \hat{\mathbf{n}}(s) \cdot \mathbf{e}, \quad \text { for spherical geometry. }\end{array}\right.$

$\mathbf{P}_{4}(s, 0)=\operatorname{diag}\left[S_{4}(s, 0), S_{5}(s, 0)\right]$,

where

$S_{4}(s, 0)=\frac{p_{3}^{0}(0)+p \kappa(s) I_{2}}{p_{3}^{0}(s)}+W$,

$W= \begin{cases}0, & \text { for plane geometry, } \\ \frac{I_{1}}{p_{3}^{0}(s)}, & \text { for spherical geometry, }\end{cases}$

$S_{5}(s, 0)= \begin{cases}\frac{u_{0}(0)}{u_{0}(s)}, & \text { for plane geometry, } \\ \frac{u_{0}(0) r_{0}(0)}{p} \hat{\mathbf{n}}(s) \cdot \hat{\mathbf{r}}(0), \quad \text { for spherical geometry. }\end{cases}$

However, the result in equation (20) does not specify $\mathbf{q}(s)$ completely as it ignores the component of $q(s)$ directed along the unperturbed ray at $x^{0}(s)$ which has magnitude

$\mathbf{q}(s) \cdot \dot{\mathbf{x}}^{0}(s)=S_{3}(s, 0) \delta_{n}+R_{3}(s, 0) q_{n}+Q_{3}(s, 0) q_{t}$

where

$S_{3}(s, 0)=\frac{p\left[u_{0}(s) I_{2}-I_{1}\right]}{u_{0}(s) p_{3}^{0}(s)}$

and

$R_{3}(s, 0)=\frac{p}{p_{3}^{0}(0)}\left[1-\frac{u_{0}(0)}{u_{0}(s)}-L(0) S_{3}(s, 0)\right]$,

$Q_{3}(s, 0)=1-L(0) S_{3}(s, 0)$.

This term is only negligible when the variation in $u_{0}$ along the ray path is small compared with $u_{0}$ itself. Furthermore, the result in equation (20) assumes that $q(0)$ has no component along the ray either. Such a component $q_{r}(0)$ contributes

$-L(0) S_{1}(s, 0) q_{t}(0)$ to $q_{n}(s)$ and

$\frac{p_{3}^{0}(0)}{p} R_{4}(s, 0) q_{t}(0)$

to $\dot{q}_{n}(s)$. The full solution requires a $5 \times 5$ propagator as outlined in Appendices $A$ and $C$. Nevertheless, those concerned only with amplitude calculations are often able to work exclusively with just the transverse components of $q(s)$ and $\delta(s)$ (see Section 4.4). Whichever form of the ray propagator for the unperturbed medium with depth-varying velocity field is used, only two integrals need to be evaluated along the ray; the propagator is completely specified by the values of these two integrals together with local values of the velocity field, its gradient and the ray direction at either end of the ray path.

$\Pi$ may be used in the two-point ray tracing problem in the unperturbed velocity field to obtain an estimate of the corrections required to the initial conditions of the unperturbed ray in order to obtain the endpoint of the ray at a particular location. For example, suppose we wish to estimate the change in $\varepsilon \delta(0)$ required to alter the endpoint of the ray by an amount $\varepsilon \xi$ whilst retaining the same initial point. When $\xi(s)$ is transverse to the ray, we may use equation (20) to obtain

$\boldsymbol{\xi}(s)=\mathbf{P}_{1}(s, 0) \mathbf{q}(0)+\mathbf{P}_{2}(s, 0) \boldsymbol{\delta}(0)$

with $\mathbf{q}(0)=\mathbf{0}$. Thus

$\delta(0)=\mathbf{P}_{2}^{-1}(s, 0) \xi(s)$,

provided $\mathbf{P}_{2}$ is non-singular.

Of course, this is a linearized approximation to the change in $\delta(0)$ required and its accuracy will depend on the size of $\varepsilon \xi(s)$-therefore some iteration may be required. This sort of calculation might prove useful in the ray shooting part of a generalized linear inversion technique where the forward modelling procedure uses the unperturbed ray field with corrections to account for any lateral variations in the velocity field. However, the effect on the properties of the perturbed ray produced by such a small change to the unperturbed ray is not so simply expressed, as we shall see in Section 4.

Finally, we take $f_{1} \neq 0$ in the solution to equation (18) given in Appendices $A$ and $C$ to find expressions for the first-order corrections to ray geometry. For zero initial conditions, the transverse components of $x^{\varepsilon}(s)$ are given by

$\mathbf{x}_{7}^{\varepsilon}(s)=\int_{0}^{s} \mathbf{P}_{2}(s, \sigma) \nabla^{T} f_{1}\left[\mathbf{x}^{0}(\sigma)\right] d \sigma$,

whilst the component of $x^{*}(s)$ directed along the unperturbed ray at $x^{0}(s)$ is

$\mathbf{x}^{\varepsilon}(s) \cdot \dot{\mathbf{x}}^{\mathrm{O}}(s)=\int_{0}^{s} S_{3}(s, \sigma) \nabla_{n}^{\mathrm{T}} f_{1}\left[\mathbf{x}^{\mathrm{o}}(\sigma)\right] d \sigma$,

where $\nabla_{n}^{T} \equiv \hat{\mathbf{n}}(s) \cdot \nabla^{T}$.

Neglect of this tangential component of $\mathbf{x}^{\varepsilon}$ requires an assumption that $\boldsymbol{u}_{0}$ does not vary greatly along the ray path.

The component of $\dot{\mathbf{x}}^{\varepsilon}(s)$ directed along the unperturbed ray is zero allowing $\dot{\mathbf{x}}^{E}(s)$ to be expressed completely in terms of the transverse ray components in the $n-$ and $x_{2}-$ 
(or $\phi-$ ) directions:

$$
\dot{\mathbf{x}}^{\varepsilon}(s)=\int_{0}^{s} \mathbf{P}_{4}(s, \sigma) \nabla^{T} f_{1}\left[\mathbf{x}^{o}(\sigma)\right] d \sigma .
$$

Thus we may write

$$
\left(\begin{array}{c}
\mathbf{x}_{T}^{\varepsilon} \\
\dot{\mathbf{x}}^{\varepsilon}
\end{array}\right)=\int_{0}^{s} \Pi(s, \sigma)\left(\begin{array}{c}
0 \\
\nabla^{T} f_{1}\left[\mathbf{x}^{0}(\sigma)\right]
\end{array}\right) d \sigma .
$$

The more general solution to equation (18) given in Appendices $A$ and $C$ gives insight on the significance of the weighting factors which multiply $\nabla^{T} f_{1}\left[x^{0}(\sigma)\right]$ in the expressions for $x^{E}$ and $\dot{x}^{E}$. It is clear from the form of solution that the weighting factors for the various components of $\nabla^{T} f_{1}\left[\mathbf{x}^{0}(\sigma)\right]$ basically described the effect of a small change in direction of the ray at the point $x^{0}(\sigma)$ as it would be observed at the point $\mathbf{x}^{0}(s)$. Thus the integrals for $\mathbf{x}^{\varepsilon}$ and $\dot{x}^{\varepsilon}$ actually describe the cumulative effect of all the 'deflections' produced by $\nabla^{T} f_{1}$ along the ray path. It is interesting to note that these ' $S$-factors' also occur as part of the definition of the corresponding ' $R$-factor' which describes the effect of a small change in the initial position of the ray; each time the $S$-factor is multiplied by $-p L(0) / p_{3}^{0}(0)$ and thus describes the deflection associated with the initial curvature of the ray.

\section{WEAK LATERAL VELOCITY INHOMOGENEITIES SUPERIMPOSED ON CONSTANT GRADIENT OF QUADRATIC SLOWNESS}

As noted by Červený (1987), the simplest analytical solution for any type of inhomogeneous medium at all is probably the polynomial solution obtained for the case of constant gradient of the quadratic slowness. If the quadratic slowness is a linear function of the Cartesian coordinate $x_{3}$ :

$\left[u_{0}\left(x_{3}\right)\right]^{2}=A+B x_{3}$,

then the ray tracing system (7) with appropriate initial conditions yields the following exact polynomial solution.

$\mathbf{x}^{0}(w)=\mathbf{x}^{\theta}\left(w_{0}\right)+\mathbf{p}^{0}\left(w_{0}\right)\left(w-w_{0}\right)+\frac{B}{4}\left(w-w_{0}\right)^{2} \mathbf{k}$,

$\mathbf{p}^{0}(w)=\mathbf{p}^{0}\left(w_{0}\right)+\frac{B}{2}\left(w-w_{0}\right) \mathbf{k}$,

where

$w=w_{0}+\int_{0}^{s} \frac{d s^{\prime \prime}}{u_{0}\left(s^{\prime \prime}\right)}$.

Thus

$w-w_{0}=\frac{x_{1}^{0}(s)-x_{1}^{0}(0)}{p}$.

For this particular geometry of the unperturbed ray, it is possible to calculate analytically the ray integrals $I_{1}$ and $I_{2}$ given at equation (22). It is found that

$$
\begin{aligned}
\kappa(s) & =\frac{B}{2} \frac{p}{\left[u_{0}(s)\right]^{3}}, \\
I_{1}= & \frac{2}{B}\left[p^{2}+p_{3}^{0}(0) p_{3}^{0}(s)\right]\left[p_{3}^{0}(s)-p_{3}^{0}(0)\right], \\
I_{2}= & \frac{2}{B}\left[p_{3}^{0}(0) p_{3}^{0}(s) \ln \left|\frac{u_{0}(s)+p_{3}^{0}(s)}{u_{0}(0)+p_{3}^{0}(0)}\right|\right. \\
& \left.+u_{0}(0) p_{3}^{0}(s)-u_{0}(s) p_{3}^{0}(0)\right]
\end{aligned}
$$

Clearly, none of these expressions becomes singular at a turning point of the unperturbed ray. Furthermore, it may be shown that the combination of terms in each element of $\Pi$ is such that none of those elements becomes singular at a turning point either. To simplify the expressions for the various elements of $\Pi$ in the case of weak lateral velocity inhomogeneities superimposed on constant gradient of quadratic slowness, it is convenient to write

$F=\ln \left|\frac{u_{0}(s)+p_{3}^{0}(s)}{u_{0}(0)+p_{3}^{0}(0)}\right|$.

Then

$$
\begin{aligned}
S_{3}(s, 0)= & \left\{\begin{aligned}
\frac{2}{B} \frac{p}{u_{0}(s)}\left\{u_{0}(s)\left[p_{3}^{0}(0) F+u_{0}(0)\right]\right. \\
\left.+\left[p_{3}^{0}(0)\right]^{2}-p^{2}-2 p_{3}^{0}(0) p_{3}^{0}(s)\right\}, \quad B \neq 0 \\
0, \quad B=0
\end{aligned}\right. \\
S_{4}(s, 0)= & \frac{1}{\left[u_{0}(s)\right]^{3}}\left\{p^{2}\left\{p_{3}^{0}(0) F+u_{0}(0)\right]\right. \\
& \left.+u_{0}(s) p_{3}^{0}(s) p_{3}^{0}(0)\right\} \\
R_{1}(s, 0)= & \frac{1}{u_{0}(s)\left[u_{0}(0)\right]^{3}}\left\{p^{4}+p_{3}^{0}(s) p_{3}^{0}(0)\right. \\
& \left.\times\left[2 p^{2}+\left[u_{0}(0)\right]^{2}\right]-p^{2}\left[p_{3}^{0}(s)\right]^{2}\right\} \\
R_{3}(s, 0)= & \frac{p}{u_{0}(s)\left[u_{0}(0)\right]^{3}}\left\{2 p^{2}\left[p_{3}^{0}(s)-p_{3}^{0}(0)\right]\right. \\
& \left.+u_{0}(0) p_{3}^{0}(0)\left[u_{0}(s)-u_{0}(0)\right]-p^{2} u_{0}(s) F\right\} \\
R_{4}(s, 0)= & \frac{B}{2} \frac{p^{2}}{\left[u_{0}(s) u_{0}(0)\right]^{3}} \\
& \times\left\{u_{0}(0) p_{3}^{0}(0)-u_{0}(s) p_{3}^{0}(s)-p^{2} F\right\} .
\end{aligned}
$$

Thus, all terms in $\Pi$ may be expressed simply in terms of the values of $x_{1}^{0}, u_{0}$ and $p_{3}^{0}$ at both the beginning and end of the corresponding unperturbed ray path and no numerical integration is required beyond the calculation of $w(s)$. The $S$-factors appear as the weighting factors of the various components of $\nabla^{T} f_{1}\left[\mathbf{x}^{0}(\sigma)\right]$ in the integrals for the deviation in ray geometry produced by weak lateral inhomogeneities superimposed on the constant gradient of quadratic slowness; again, there are no singularities associated with the turning points of the unperturbed rays.

\section{PERTURBATIONS IN THE PROPERTIES OF THE RAY}

We now consider rays passing through a plane or spherical layer of thickness $h$ with slowness field given by equation (4) 
and whose trajectories prior to their impinging on this layer are well-defined. In the absence of perturbations, it would be the ray through the point $P$ on the base of the layer which would arrive at $O$ on the surface (see Fig. 2). We suppose that the ray has travelled for a time $\tau_{0}(\mathrm{P})$ from the source to $P$ and take as our reference surface the unperturbed wavefront of the disturbance at $P$, that is, $\tau(\mathbf{x})=\tau_{0}(P)$. We may introduce orthogonal ray coordinates $\xi, \eta$ on this surface ( $c f$. Appendix D) such that $\mathbf{P}$ has coordinates $\xi_{0}, \eta_{0}$ on the surface. The associated scale factors and unit vectors are defined in equations (D-1). In view of the geometry outlined in Section 2 , it would seem that the appropriate choice for these transverse ray coordinates would be to take $\xi$ as the 2-coordinate in the vertically varying medium and the $\phi$-coordinate in the spherically symmetric medium with $\eta$ as the $n$-coordinate as defined previously for the two types of media.

However, as indicated in Fig. 2, it is not the ray through $\mathbf{P}$ which arrives at $O$ on the surface when there are perturbations in the velocity field of the layer but another ray-say, the ray through $\left[\xi_{0}+\varepsilon \xi_{\varepsilon}+0\left(\varepsilon^{2}\right), \eta_{0}+\varepsilon \eta_{\varepsilon}+\right.$ $\left.0\left(\varepsilon^{2}\right)\right]$. The point at which this ray enters the layer is denoted by $\mathbf{P}^{\prime}$ and, generally speaking, $\mathbf{P}^{\prime}$ will lie on a different wavefront from $P$. In the absence of perturbations, this ray through $\mathbf{P}^{\prime}$ would arrive at the surface $\mathrm{O}^{\prime}$. From this brief consideration of Fig. 2, it is clear that the fluctuations in the velocity field produce corresponding variations in the curvilinear coordinates of points in space. Now in equations (9.1)-(9.5) of paper I, it was shown that the first-order corrections to the curvilinear coordinates of an arbitrary point previously described by its location $x^{0}\left(s_{0}, \xi_{0}, \eta_{0}\right)$ on the unperturbed ray are given by $\varepsilon \xi_{\varepsilon}, \varepsilon \eta_{\varepsilon}$ and $\varepsilon s_{\varepsilon}$ where

$\xi_{\boldsymbol{E}}=-\frac{\mathbf{e}_{\xi} \cdot \mathbf{x}^{\varepsilon}\left(s_{0}, \xi_{0}, \eta_{0}\right)}{h_{\xi}}$,

$\eta_{\varepsilon}=-\frac{\mathbf{e}_{\eta} \cdot \mathbf{x}^{\varepsilon}\left(s_{0}, \xi_{0}, \eta_{0}\right)}{h_{\eta}}$,

$s_{\varepsilon}=-\mathbf{e}_{s} \cdot \mathbf{x}^{\varepsilon}\left(s_{0}, \xi_{0}, \eta_{0}\right)$,

with the unit vectors and scale factors also evaluated at $\left(s_{0}, \xi_{0}, \eta_{0}\right)$. Occasionally, it is advantageous to use traveltime $\tau$ rather than arclength $s$ as the coordinate along the ray. In that case, the first-order correction to the
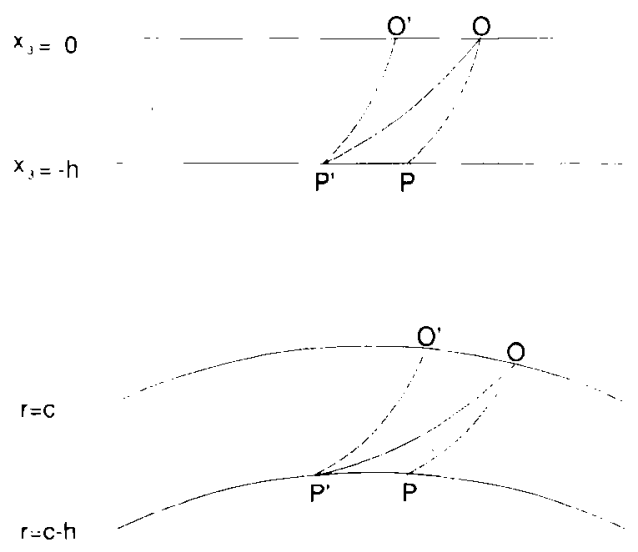

Figure 2. The perturbations in the velocity field of the layer cause the ray through $P^{\prime}$ to arrive at the surface receiver $O$, rather than the ray through $P$. coordinate along the ray is $\varepsilon \tau_{\varepsilon}$ where

$\tau_{\varepsilon}=\frac{\mathbf{e}_{\tau} \cdot \mathbf{x}^{\varepsilon}\left(\tau_{0}, \xi_{0}, \eta_{0}\right)}{h_{\tau}}=-\mathbf{p}^{0} \cdot \mathbf{x}^{\varepsilon}\left(\tau_{0}, \xi_{0}, \eta_{0}\right)$

and $\mathbf{p}^{0}=u_{0} d \mathbf{x}^{0} / d s$, the slowness vector on the unperturbed ray with all quantities evaluated at $\left(\tau_{0}, \xi_{0}, \eta_{0}\right)$. Thus $\tau_{\varepsilon}$ and $s_{\varepsilon}$ differ only by the scale factor $h_{\mathbf{t}}$. In the remaining calculations of this section, equations (38) and (39) will be applied at the surface receiver location; careful consideration will also be given to the implications of the changed path of integration where appropriate. We now consider the effects of these velocity perturbations on various observable quantities associated with the propagation of the ray through the layer.

\subsection{Traveltime perturbation}

The time taken for the unperturbed ray through $P$ to travel from the source to surface receiver at $O$ is

$t=\tau_{0}(\mathrm{P})+\int_{s_{0}(\mathrm{P})}^{s_{0}(\mathrm{O})} u_{0}\left(\mathbf{x}^{0}\right) d s=\tau_{0}(\mathrm{O})$,

where the argument of the integrand indicates that the path of integration is along $x^{0}(s)$, the unperturbed ray path from $\mathbf{P}$ to $O$. The time taken for the perturbed ray through $\mathbf{P}^{\prime}$ to travel from the source to surface received at $O$ is

$t^{\prime}=\tau_{0}\left(\mathrm{P}^{\prime}\right)+\int_{s_{0}\left(\mathrm{P}^{\prime}\right)}^{s(\mathrm{O})}\left[1+\varepsilon f_{1}(\mathbf{x})\right] u_{0}(\mathbf{x}) d s+0\left(\varepsilon^{2}\right)$

where the argument of the integrand now indicates that the path of integration is along $\mathbf{x}(s)$ which is given by equation (6); the upper limit of integration is given by

$s(\mathrm{O})=s_{0}(\mathrm{O})+\varepsilon s_{\varepsilon}(\mathrm{O})+0\left(\varepsilon^{2}\right)$,

where

$s_{\varepsilon}(\mathrm{O})=-\mathbf{e}_{\mathrm{s}}^{0} \cdot \mathbf{x}^{\varepsilon}\left[s_{0}(\mathrm{O}), \xi_{0}, \eta_{0}\right]$,

and $\mathbf{e}_{\mathrm{a}}^{0}$ denotes the direction at which the unperturbed ray would arrive at $\mathrm{O}$.

In general, the path length $s(\mathrm{O})$ is different from $s_{0}\left(\mathrm{O}^{\prime}\right)$, the path length to the surface along the ray $\mathrm{P}^{\prime} \mathrm{O}^{\prime}$ when there are no perturbations.

Now, as $u_{0}(\mathbf{x})$ is a smoothly varying function of position and $\varepsilon \mathbf{x}^{\varepsilon}$ is small,

$u_{0}(\mathbf{x}) \sim u_{0}\left(\mathbf{x}^{0}+\varepsilon \mathbf{x}^{\varepsilon}\right) \sim u_{0}\left(\mathbf{x}^{0}\right)+\varepsilon \mathbf{x}^{\varepsilon} \cdot \nabla u_{0}\left(\mathbf{x}^{0}\right)$

and so

$$
\begin{aligned}
\int_{s_{0}\left(\mathrm{P}^{\prime}\right)}^{s(\mathrm{O})} u_{0}(\mathbf{x}) d s= & \int_{s_{0}\left(\mathrm{P}^{\prime}\right)}^{s(\mathrm{O})} u_{0}\left(\mathbf{x}^{\mathrm{O}}\right) d s \\
& +\varepsilon \int_{s_{0}\left(\mathrm{P}^{\prime}\right)}^{s(\mathrm{O})} \mathbf{x}^{\varepsilon} \cdot \nabla u_{0} d s+0\left(\varepsilon^{2}\right),
\end{aligned}
$$

where both integrals on the right are now evaluated along the unperturbed ray path through $\mathrm{P}^{\prime}$, which is different from the path of integration in equation (40).

Using equation (1) and integrating by parts gives

$$
\int_{s 0\left(\mathrm{P}^{\prime}\right)}^{s(\mathrm{O})} u_{0}(\mathbf{x}) d s=\int_{s_{0}\left(\mathrm{P}^{\prime}\right)}^{s(\mathrm{O})} u_{0}\left(\mathbf{x}^{\mathrm{o}}\right) d s+\varepsilon\left[\mathbf{x}^{\varepsilon} \cdot \mathbf{P}^{0}\right]_{s_{0}\left(\mathrm{P}^{\prime}\right)}^{s(\mathrm{O})}+0\left(\varepsilon^{2}\right),
$$


as

$\frac{d \mathbf{x}^{\varepsilon}}{d s} \cdot \mathbf{p}^{0}=0$

at all points along the ray by equations (A-17) and (C-4).

Furthermore, by hypothesis, $\mathbf{x}^{\varepsilon}\left[s_{0}\left(\mathrm{P}^{\prime}\right)\right]=\mathbf{0}$ and so

$$
\begin{aligned}
\int_{s_{o}\left(\mathbf{P}^{\prime}\right)}^{s(\mathrm{O})} u_{0}(\mathbf{x}) d s= & \int_{s_{0}\left(\mathbf{P}^{\prime}\right)}^{s(\mathrm{O})} u_{0}\left(\mathbf{x}^{0}\right) d s \\
& +\varepsilon \mathbf{x}^{\varepsilon}\left[s_{0}(\mathrm{O})\right] \cdot \mathbf{p}^{0}\left[s_{0}(\mathrm{O})\right]+0\left(\varepsilon^{2}\right),
\end{aligned}
$$

as first-order increments in the arguments of the second term contribute only terms $0\left(\varepsilon^{2}\right)$.

Now, using the notation of equation (39) in equation (46), we obtain from equation (41)

$$
\begin{aligned}
t^{\prime}= & \tau_{0}[s(\mathrm{O}), \xi, \eta]-\varepsilon \tau_{\varepsilon}\left[s_{0}(\mathrm{O})\right] \\
& +\varepsilon \int_{s_{0}(\mathbf{P})}^{s(\mathrm{O})} u_{0}\left(\mathbf{x}^{0}\right) f_{1}\left(\mathbf{x}^{0}\right) d s+0\left(\varepsilon^{2}\right)
\end{aligned}
$$

as changing the path of integration in the third term of equation (47) contributes only terms $0\left(\varepsilon^{2}\right)$. On the other hand, Taylor expansion of $\tau_{0}[s(O), \xi, \eta]$ about its value at $\left[s_{0}(\mathrm{O}), \xi_{0}, \eta_{0}\right]$ gives

$$
\begin{aligned}
\tau_{0}\left[s_{0}(\mathrm{O}), \xi, \eta\right]= & \tau_{0}\left[s_{0}(\mathrm{O}), \xi_{0}, \eta_{\mathrm{o}}\right] \\
& -\left.\varepsilon \mathbf{x}^{\varepsilon} \cdot \nabla \tau_{0}\right|_{s_{0}(O), \xi_{0}, \eta_{0}}+0\left(\varepsilon^{2}\right) \\
= & \tau_{0}\left[s_{0}(\mathrm{O}), \xi_{0}, \eta_{\mathrm{o}}\right] \\
& +\varepsilon \tau_{\varepsilon}\left[s_{0}(\mathrm{O}), \xi_{0}, \eta_{\mathrm{o}}\right]+0\left(\varepsilon^{2}\right)
\end{aligned}
$$

Therefore,

$t^{\prime}-t=\varepsilon t^{\varepsilon}+0\left(\varepsilon^{2}\right), \quad \tau^{\varepsilon}=\int_{s_{0}(\mathbf{P})}^{s_{0}(O)} u_{0}\left(\mathbf{x}^{0}\right) f_{1}\left(\mathbf{x}^{0}\right) d s$.

That is, the perturbations in the traveltime are given by the same equation as in paper $I$, although the path of integration varies in accordance with the different unperturbed ray geometries. Furthermore, equation (49) is equivalent to the linear functional obtained by Johnson \& Gilbert (1972) in their linearization of the inverse problem for traveltimes by using the concept of Fréchet differentiability.

\subsection{Slowness perturbations}

The direction of approach to the surface of the unperturbed ray through $\mathrm{P}$ is given by

$\mathrm{d}^{0}=\frac{d \mathbf{x}^{0}}{d s}(\mathrm{O})$

where the argument of the function denotes evaluation at the surface receiver $O$. However, for the perturbed ray through $P^{\prime}$, the direction of approach to the surface is given by

$$
\begin{aligned}
\mathbf{d}= & \left\{\frac{d \mathbf{x}^{0}}{d s}+\varepsilon\left[\frac{d \mathbf{x}^{\varepsilon}}{d s}+s_{\varepsilon} \frac{\partial}{\partial s}\left(\frac{d \mathbf{x}^{0}}{d s}\right)\right.\right. \\
& \left.\left.+\xi_{\varepsilon} \frac{\partial}{\partial \xi}\left(\frac{d \mathbf{x}^{0}}{d s}\right)+\eta_{\varepsilon} \frac{\partial}{\partial \eta}\left(\frac{d \mathbf{x}^{0}}{d s}\right)\right]\right\}+0\left(\varepsilon^{2}\right),
\end{aligned}
$$

which may be simplified using equation (38) to obtain

$\mathbf{d}=\left\{\frac{d \mathbf{x}^{0}}{d s}+\varepsilon\left[\frac{d \mathbf{x}^{\varepsilon}}{d s}-\mathbf{x}^{\varepsilon} \cdot \nabla\left(\frac{d \mathbf{x}^{0}}{d s}\right)\right]\right\}+0\left(\varepsilon^{2}\right)$.

Thus, the variation in the surface direction of approach of the rays resulting from the fluctuations in the seismic velocity field is

$\mathbf{d}-\mathbf{d}^{0}=\varepsilon \mathbf{d}^{\varepsilon}+0\left(\varepsilon^{2}\right), \quad \mathbf{d}^{\varepsilon}=\frac{d \mathbf{x}^{\varepsilon}}{d s}-\mathbf{x}^{\varepsilon} \cdot \nabla\left(\frac{d \mathbf{x}^{0}}{d s}\right)$.

This expression is in agreement with the corresponding result in paper $I$ for the case $\alpha=$ constant, although in that case different notation was used because the variation in $d \mathbf{x}^{0} / d s$ was expressed in terms of the deviation in the point of entry to the heterogeneous layer rather than in terms of surface quantities. An expression for $\nabla\left(d x^{\circ} / d s\right)$ is derived at the end of Appendix $D$; using the notation $\left(d x_{i}^{0} / d s\right)_{, k}$ for this quantity, it is found that there is no $i$-component along the ray and so when we contract with $x^{\varepsilon}$, we find that the component of $\mathrm{d}^{\varepsilon}$ in the direction of $\mathrm{d}^{0}$ is zero. When $d \mathrm{x}_{0} / d s$ does not change significantly across the wavefront, (D-15) may be used in (52) to obtain

$\mathrm{d}^{\varepsilon}=\frac{d \mathrm{x}^{\varepsilon}}{d s}-x_{r} \kappa(s) \hat{\mathbf{n}}=\left(\begin{array}{c}\dot{x}_{n}^{\varepsilon} \\ \dot{x}_{2}^{\varepsilon}\end{array}\right)$.

Otherwise, $d_{n}^{\varepsilon}$ is increased by $\left(\partial \ln h_{\eta} / \partial s\right) x_{n}^{\varepsilon}$. However, when $h_{\eta}=1$ everywhere on the unperturbed ray this increment vanishes and the result in equation (53) still holds.

Now, from a phenomenological viewpoint (cf. section 7, paper I) we would expect the first-order perturbation in the surface slowness to be given by

$\mathbf{P}^{\varepsilon}=u_{0}(\mathrm{O})\left[f_{1}(\mathrm{O}) \frac{d \mathbf{x}^{\circ}}{d s}+\mathbf{d}^{\varepsilon}\right]$

where $d^{E}$ is given by equation (52) and all quantities are evaluated at the surface receiver $O$. In fact, it is also possible to derive this result by evaluating the gradient of traveltime curve using the results of Section 4.1. Thus, with the expressions for $\mathrm{x}^{\varepsilon}$ and $d \mathrm{x}^{\varepsilon} / d s$ given in Section 2 , it follows that the transverse components of the variation in surface slowness resulting from the perturbations in the seismic velocity field depend only on the variation of $\nabla^{T} f_{1}$ throughout the layer.

\subsection{Amplitude fluctuations}

The expression for the amplitude fluctuation is again derived from equation (8.9) of paper I. As before, the area of surface element on the wavefront is denoted by

$J=\left|\frac{\partial \mathbf{x}}{\partial \xi} \times \frac{\partial \mathbf{x}}{\partial \eta}\right|=h_{\xi} h_{\eta}$

In this context, it seems preferable to use $\tau$ rather than $s$ as the coordinate along the ray; then the amplitude is given by the relation

$\Phi(\tau, \xi, \eta)=\left.A^{0}(\xi, \eta) \sqrt{\frac{J_{0} \rho^{0}}{u_{0}} \cdot \frac{u}{\rho h_{\xi} h_{\eta}}}\right|_{\tau, \xi, \eta}$, 
where $A^{0}(\xi, \eta)$ denotes the amplitude distribution across the wavefront at some reference level; and $\rho_{0}, u_{0}$ and $J_{0}$ respectively denote the values of the density, slowness field and area of surface element on the wavefront at the reference point.

In fact, the expression for amplitude with $s$ as the coordinate along the ray would have essentially the same form as (56) although $A^{\circ}(\xi, \eta)$ would no longer denote the amplitude distribution across a wavefront ( $\tau=$ const.) but across some surface in space defined by the equation $s=$ constant; this seems a less natural choice. Either way, the derivation of the expression for fluctuation in amplitude resulting from perturbations in the velocity field is similar to that given in section 9 of paper I except that it is now necessary to make allowance for the fact that $\tau_{\varepsilon}$ (or $s_{\varepsilon}$ ) is no longer zero and that $h_{\tau}$ (or $h_{s}$ ) may vary across the wavefront. Neverthelsss, from equations (38) and (39) we have

$\tau_{\varepsilon} \frac{\partial \Phi_{0}}{\partial \tau}+\xi_{\varepsilon} \frac{\partial \Phi_{0}}{\partial \xi}+\eta_{\varepsilon} \frac{\partial \Phi_{0}}{\partial \eta}=-\mathbf{x}^{\varepsilon} \cdot \nabla \Phi_{0}$

$s_{\varepsilon} \frac{\partial \Phi_{0}}{\partial s}+\xi_{\varepsilon} \frac{\partial \Phi_{0}}{\partial \xi}+\eta_{\varepsilon} \frac{\partial \Phi_{0}}{\partial \eta}=-\mathbf{x}^{\varepsilon} \cdot \nabla \Phi_{0}$

Thus, the results can be stated without explicit reference to $\tau_{\varepsilon}\left(\right.$ or $s_{\varepsilon}$ ).

Now on the perturbed ray,

$$
\begin{aligned}
J[\mathbf{x}(\tau, \xi, \eta)] & =\left|\left(h_{\xi} \mathbf{e}_{\xi}+\varepsilon \frac{\partial \mathbf{x}^{\varepsilon}}{\partial \xi}\right) \times\left(h_{\eta} \mathbf{e}_{\eta}+\frac{\partial \mathbf{x}^{\varepsilon}}{\partial \eta}\right)\right|+0\left(\varepsilon^{2}\right) \\
& =h_{\xi} h_{\eta}\left[1+\varepsilon\left(\frac{\mathbf{e}_{\xi}}{h_{\xi}} \cdot \frac{\partial \mathbf{x}^{\varepsilon}}{\partial \xi}+\frac{\mathbf{e}_{\eta}}{h_{\eta}} \cdot \frac{\partial \mathbf{x}^{\varepsilon}}{\partial \eta}\right)\right]+0\left(\varepsilon^{2}\right)
\end{aligned}
$$

and thus the expression for the perturbed amplitude is simply

$$
\begin{aligned}
\Phi= & \Phi_{0}\left(\tau_{0}, \xi_{0}, \eta_{0}\right)\left\{1-\frac{\varepsilon}{2}\left(\mathbf{x}^{\varepsilon} \cdot \mathbf{v}+\nabla \cdot \mathbf{x}_{T}^{\varepsilon}\right)\right. \\
& \left.+\frac{\varepsilon}{2}\left[f_{1}(\mathrm{O})-f_{2}(\mathrm{O})\right]-\frac{\varepsilon}{2}\left[f_{1}(\mathrm{P})-f_{2}(\mathrm{P})\right]\right\}+0\left(\varepsilon^{2}\right)
\end{aligned}
$$

where

$\rho(\mathbf{x})=\rho_{0}\left[1+\varepsilon f_{2}(\mathbf{x})\right]$,

$\mathbf{x}_{T}^{\varepsilon}=\mathbf{x}^{\varepsilon}-\left(\mathbf{x}^{\varepsilon} \cdot \mathbf{e}_{\tau}\right) \mathbf{e}_{\tau}=\left(\mathbf{x}^{\varepsilon} \cdot \mathbf{e}_{\xi}\right) \mathbf{e}_{\xi}+\left(\mathbf{x}^{\varepsilon} \cdot \mathbf{e}_{\eta}\right) \mathbf{e}_{\eta}$,

and

$\mathbf{v}=\nabla \ln \Phi_{0}^{2}+\gamma$

with

$\gamma=-\left(h_{\tau} h_{\xi} h_{\eta}\right)^{-1}\left[\frac{\partial}{\partial \xi}\left(h_{\tau} h_{\eta} \mathbf{e}_{\xi}\right)+\frac{\partial}{\partial \eta}\left(h_{\tau} h_{\xi} \mathbf{e}_{\eta}\right)\right]$.

$\gamma$ represents the component of $\mathbf{v}$ resulting from the change in ray geometry produced by the fluctuations in the seismic velocity field whereas $\nabla \ln \Phi_{0}^{2}$ takes account of the fact that it is a different ray arriving at the point of observation and that the amplitude distribution may vary slightly across the wavefront. This distinction will be important in the study of the fluctuation in polarization for $S$-waves in the next subsection.

In the ray coordinates introduced previously, it may be shown that

$\gamma=\kappa(s) \hat{\mathbf{n}}+\frac{\partial \ln J}{\partial s} \mathbf{t}$.

From equation (59), it is seen that the amplitude fluctuations depend on both $x^{\varepsilon}$ and the divergence of its transverse components at the point of observation as well as the local fluctuations in the velocity and density fields at the points where the ray enters and leaves the scattering layer. From a computational point of view, it would seem that the easiest way to calculate $\nabla \cdot \mathbf{x}_{T}^{\varepsilon}$ would be to trace two nearby rays (with slight displacements in the $\xi$ and $\eta$ directions respectively) and compute the corresponding changes in $\mathbf{x}_{T}^{\varepsilon}$ to use in a quotient with appropriate components of the resulting changes in $x^{0}$ on the nearby rays.

Alternatively, it may be shown that when $\xi$ and $\eta$ are the transverse ray coordinates outlined previously and $h_{\eta}=1$ everywhere on the unperturbed ray, then

$\frac{\partial S_{1}}{\partial n}=\frac{1}{u_{0}(s)} \int_{\sigma}^{s}\left[\kappa(\sigma)-\kappa\left(s^{\prime \prime}\right)\right] \frac{p_{3}^{0}(s) p_{3}^{0}(\sigma) u_{0}\left(s^{\prime \prime}\right)}{\left[p_{3}^{0}\left(s^{\prime \prime}\right)\right]^{2}} d s$

and

$$
\begin{aligned}
\nabla \cdot \mathbf{x}_{r}^{\varepsilon}= & \int_{0}^{s} \frac{\partial S_{1}}{\partial n} \nabla_{r}^{T} f_{1}\left[\mathbf{x}^{0}(\sigma)\right] d \sigma \\
& +\int_{0}^{s} \mathbf{P}_{2}\left(\nabla^{T}\right)^{2} f_{1}\left[\mathbf{x}^{0}(\sigma)\right] d \sigma
\end{aligned}
$$

\subsection{Fluctuation in polarization (for $S$-waves)}

The unit vectors $\mathrm{e}^{S V}, \mathrm{e}^{S H}$ which define the orientation of the $S V$ - and $S H$-components of the displacement field at the surface of the layer in the absence of perturbations in the velocity field may be written in the form

$\mathrm{e}^{S v}=\frac{v-\left(v \cdot \mathrm{d}^{0}\right) \mathrm{d}^{0}}{\sqrt{1-\left(v \cdot \mathrm{d}^{0}\right)^{2}}}, \quad \mathrm{e}^{S H}=\frac{v \times \mathrm{d}^{0}}{\sqrt{1-\left(v \cdot \mathrm{d}^{0}\right)^{2}}}$,

where $\mathrm{d}^{0}$ is given by equation (50); and

$\boldsymbol{v}= \begin{cases}\mathbf{k}, & \text { for plane geometry, } \\ \hat{\mathbf{r}}, & \text { for spherical geometry. }\end{cases}$

Of course, in the ray coordinates introduced previously,

$\mathbf{e}^{S V}=\hat{\mathbf{n}}$ and $\mathbf{e}^{S H}= \begin{cases}\mathbf{j}, & \text { for plane geometry, } \\ \hat{\boldsymbol{\theta}}, & \text { for spherical geometry }\end{cases}$

However, if in equation (64) we replace $d^{0}$ by $\mathbf{d}=\mathbf{d}^{0}+\varepsilon \mathbf{d}^{\varepsilon}+0\left(\varepsilon^{2}\right)$, we obtain the variation in the directions of $\mathrm{e}^{S V}$ and $\mathrm{e}^{S H}$ which result from the fluctuations in the velocity field; they are respectively

$\frac{\varepsilon d_{3}^{0} d_{3}^{\varepsilon}}{1-d_{3}^{02}} \mathbf{e}^{s V}-\frac{\varepsilon\left(d_{3}^{\varepsilon} \mathbf{d}^{0}+d_{3}^{0} \mathbf{d}^{\varepsilon}\right)}{\sqrt{1-d_{3}^{02}}}+0\left(\varepsilon^{2}\right)$

and

$\frac{\varepsilon d_{3}^{0} d_{3}^{\epsilon}}{1-d_{3}^{02}} \mathbf{e}^{S H}+\frac{\varepsilon \boldsymbol{v} \times \mathbf{d}^{\varepsilon}}{\sqrt{1-d_{3}^{02}}}+0\left(\varepsilon^{2}\right)$

where the subscript 3 denotes the $v$-component as before.

Now, we suppose that in the absence of perturbations, the 
surface $S$-displacement field may be expressed as

$\mathbf{U}^{0}=E_{1}^{0} \mathbf{e}^{S V}+E_{2}^{0} \mathbf{e}^{S H}$.

Equation (65) gives the changes in the unit vectors $\mathrm{e}^{s v}$ and $\mathbf{e}^{S H}$ produced by the perturbations in the velocity field; the corresponding fluctuations in $E_{1}^{0}$ and $E_{2}^{0}$ are related to the amplitude fluctuations studied in the previous subsection, since $\Phi_{0}=\sqrt{\left(E_{1}^{0}\right)^{2}+\left(E_{2}^{0}\right)^{2}}$. Adapting equation (59) to describe amplitudes of $S$-rays, it follows that

$E_{1}=E_{1}^{0}\left[1-\varepsilon\left(\mathbf{x}^{\varepsilon} \cdot \nabla \ln E_{1}^{0}+G_{\varepsilon}\right)\right]+0\left(\varepsilon^{2}\right)$,

$E_{2}=E_{2}^{0}\left[1-\varepsilon\left(\mathbf{x}^{\varepsilon} \cdot \nabla \ln E_{2}^{0}+G_{\varepsilon}\right)\right]+0\left(\varepsilon^{2}\right)$,

where

$G_{e}=\frac{1}{2}\left\{\mathbf{x}^{\varepsilon} \cdot \gamma+\nabla \cdot \mathbf{x}_{T}^{\varepsilon}+f_{1}(\mathrm{P})-f_{2}(\mathrm{P})-\left[f_{1}(\mathrm{O})-f_{2}(\mathrm{O})\right]\right\}$.

In the perturbed medium, the components of the surface $S$-displacement field are still expressed relative to the unit vectors $\mathrm{e}_{s}, \mathrm{e}^{s V}$, and $\mathrm{e}^{S H}$ associated with the unperturbed ray geometry, that is,

$\mathbf{U}=F_{1} \mathbf{e}^{S V}+F_{2} \mathbf{e}^{S H}+F_{3} \mathbf{e}_{\mathbf{s}}$.

These components are related to the initial disturbance and the perturbed ray geometry as follows:

$F_{1}=E_{1}^{0}(1+\varepsilon l)+E_{2}^{0}(\varepsilon m)$,

$F_{2}=E_{1}^{0}(-\varepsilon m)+E_{2}^{0}[1+\varepsilon(l+\delta)]$,

$F_{3}=E_{1}^{0}(\varepsilon n)+E_{2}^{0}\left(-\sqrt{\frac{1-d_{3}^{02}}{d_{3}^{0}}} \varepsilon m\right)$,

where

$$
\begin{aligned}
& l=-\mathbf{x}^{\varepsilon} \cdot \nabla \ln E_{1}^{0}-G_{\varepsilon}-\frac{d_{3}^{0} d_{3}^{\varepsilon}}{\left(1-d_{2}^{02}\right)}, \\
& m=\frac{d_{3}^{0} \mathbf{d}^{\varepsilon} \cdot\left(v \times \mathbf{d}^{0}\right)}{\left(1-d_{3}^{0}\right)^{2}}, \quad n=\frac{-d_{3}^{\varepsilon}}{\sqrt{1-\bar{d}_{3}^{02}}}, \\
& \delta=\mathbf{x}^{\varepsilon} \cdot \nabla \ln \frac{E_{1}^{0}}{E_{2}^{0}} .
\end{aligned}
$$

The concept of Stokes' parameters from electromagnetic wave theory (Newton 1966) may be used to study the variation in polarization. Define

$I=\left|F_{1}\right|^{2}+\left|F_{2}\right|^{2}, \quad Q=\left|F_{1}\right|^{2}-\left|F_{2}\right|^{2}$
$U=-2 \mathscr{R e}_{(}\left(F_{1} F_{2}^{*}\right), \quad V=-2 \mathscr{T}_{m}\left(F_{1} F_{2}^{*}\right)$

Then from equations (69):

$$
\begin{aligned}
& I=I_{0}(1+2 \varepsilon l+\varepsilon \delta)-Q_{0} \varepsilon \delta+0\left(\varepsilon^{2}\right), \\
& Q=Q_{0}(1+2 \varepsilon l+\varepsilon \delta)-I_{0} \varepsilon \delta-2 U_{0} \varepsilon m+0\left(\varepsilon^{2}\right), \\
& U=U_{0}(1+2 \varepsilon l+\varepsilon \delta)+2 Q_{0} \varepsilon m+0\left(\varepsilon^{2}\right), \\
& V=V_{0}(1+2 \varepsilon l+\varepsilon \delta)+0\left(\varepsilon^{2}\right),
\end{aligned}
$$

where the subscript ${ }_{0}$ denotes the values of these parameters in the absence of perturbations.

$I$ represents the square of the amplitude of the $S$-motion transverse to $e_{\mathrm{s}}$ and when $F_{3}$ is zero, equation (72a) is identical to equation (59).
The polarization of the motion is given by

$$
\begin{aligned}
\tan 2\left(\Omega^{0}+\Omega^{\varepsilon}\right) & =\frac{V}{\sqrt{U^{2}+Q^{2}}} \\
& =\frac{V_{0}}{\sqrt{U_{0}^{2}+Q_{0}^{2}}}\left[1+\frac{\varepsilon \delta I_{0}}{A} \frac{Q_{0}}{\left(U_{0}^{2}+Q_{0}^{2}\right)}\right]+O\left(\varepsilon^{2}\right),
\end{aligned}
$$

where

$A=1+2 \varepsilon l+\varepsilon \delta$.

Thus

$$
\begin{aligned}
\tan 2 \Omega^{\varepsilon} & =\frac{\varepsilon \delta I_{0}}{2 A} \frac{Q_{0}}{\left(U_{0}^{2}+Q_{0}^{2}\right)} \sin 4 \Omega^{0}+0\left(\varepsilon^{2}\right) \\
& =\frac{\varepsilon \delta Q_{0}}{I_{0}} \tan 2 \Omega^{0}+0\left(\varepsilon^{2}\right),
\end{aligned}
$$

that is, the fluctuation in the polarization of $S$-waves depends on the variation in $E_{1}^{0} / E_{2}^{0}$ in the vicinity of the ray and results from the fact that the relative amplitude distributions of $S H$ - and $S V$-components may vary slightly across the wavefront. It is only when the incident $S$-wave is linearly polarized or when the ratio $E_{1}^{0} / E_{2}^{0}$ is constant across the wavefront in the vicinity of the ray of interest in the unperturbed medium, that the polarization (linear, elliptic, etc.) is unaltered by the variations in the velocity field. Even slight variations in $E_{1}^{0} / E_{2}^{0}$ across the wavefront in the vicinity of the ray of interest can produce significant changes in polarization when $2 \Omega^{0} \approx \pi / 2$ (circular polarization).

\section{CONCLUSIONS}

The effect of small arbitrary perturbations in the seismic velocity field of a layer of fixed thickness in which the unperturbed velocity field varies with depth has been studied for both plane and spherical geometries. Assuming that the trajectories of the rays in the unperturbed medium are known, it has been possible to derive expressions for the perturbations in the ray geometry which result from these fluctuations in the seismic velocity field. It is found that although $d \mathrm{x}^{\varepsilon} / d s$ is perpendicular to $d \mathrm{x}^{0} / d s$ at each point along the ray, the displacement vector $x^{\varepsilon}(s)$ may indeed have a component directed along the ray at the point of observation-that is, it is possible for the fluctuations in the seismic velocity field to cause an apparent advance or retardation along the ray path. Nevertheless, Fermat's principle is not violated and the first-order correction to the traveltime again results purely from the fluctuations $f_{1}(x)$ in the slowness field along the unperturbed ray path.

In the course of the calculations, simple expressions are also obtained for the various components of the propagator matrices for the unperturbed ray, that is, the ray which would propagate through the prescribed depth-varying seismic velocity field (without fluctuations). These expressions require the calculation of just two integrals along the unperturbed ray path and are calculated analytically for the special case of weak lateral velocity inhomogeneities superimposed on a constant gradient of quadratic slowness. Such a propagator formalism is widely used in the study of Gaussian beams and describes how small variations in the initial position and direction of the unperturbed ray are propagated along the ray; it also gives insight into the 
significance of the weighting factors in the integral expressions for $\mathbf{x}^{\varepsilon}$ and $d \mathbf{x}^{E} / d s$.

The expressions for the first-order corrections to the direction of approach, slowness, polarization of $S$-waves and amplitude at the point of observation on the surface are all found to have essentially the same form as in paper I when expressed in terms of $x^{E}$. However, the differences in the ray geometries lead to different functional dependences on the various transverse derivatives of $f_{1}$. Nevertheless, the perturbations in the surface slowness again depend only on the variation of $\nabla^{T} f_{1}$ throughout the layer whilst the amplitude fluctuations depend also on higher derivatives of the fluctuation in the seismic velocity field throughout the layer. When the relative amplitudes of $S H$ - and $S V$-components of the motion do not vary significantly across the wavefront in the vicinity of the ray in the unperturbed medium or the incident $S$-wave is linearly polarized, the only effect of the perturbation of the seismic velocity field on the polarization of $S$-waves is to rotate the principal axes of the polarization ellipse and to first-order, this rotation depends only on the change in the direction of the ray trajectory; the relative phase of the $S H$ - and $S V$-components remains unchanged. However, when the incident $S$-wave has near-circular polarization, then even slight changes in the relative amplitudes of $\mathrm{SH}$ - and $S V$-components across the wavefront can produce significant changes in polarization. From these results it follows that the irregular spatial variations of surface slowness, amplitude and polarization for $S$-waves contain information about the structure of the seismic velocity perturbation which is additional to that contained in the traveltime residuals. The approach used in deriving these expressions gives insight into the various complicating factors which need to be considered in inverse modelling problems involving such media.

\section{REFERENCES}

Aki, K., Christoffersson, A. \& Husebye, E. S., 1977. Determination of the three-dimensional seismic structure of the lithosphere, J. geophys. Res., 82, 277-296

Červený, V., 1985. Gaussian beam synthetic seismograms, J. Geophys., 58, 44-72.

Červený, V., 1987. Ray tracing algorithms in three-dimensional laterally varying layered structures, in Seismic Tomography with Applications in Global Seismology and Exploration Geophysics, pp. 99-133, ed. Nolet, G., Reidel, Dordrecht.

Cervený, V. \& Pšencik, I., 1984. Gaussian beams in elastic two dimensional laterally varying layered structures, Geophys. J.R. astr. Soc., 78, 65-91.

Červený, V., Popov, M. M. \& Pšencík, I., 1982. Computation of wave fields in inhomogeneous media-Gaussian beam approach, Geophys. J.R. astr. Soc., 70, 109-128.

Chapman, C. H., 1978. A new method for computing synthetic seismograms, Geophys. J.R. astr. Soc., 58, 481-513.

Chapman, C. H., 1985. Ray theory and its extensions: WKBJ and Maslov seismograms, J. Geophys., 58, 27-43.

Chapman, C. H. \& Drummond, R., 1982. Body-wave seismograms in inhomogeneous media using Maslov asymptotic theory, Bull. seism. Soc. Am., 72, 5277-5317.

Farra, V. \& Madariaga, R., 1987. Seismic waveform modeling in heterogeneous media by ray perturbation theory, J. geophys. Res., 92, 2697-2712.

Johnson, L. E. \& Gilbert, F., 1972. Inversion and inference for teleseismic ray data, Methods in Computational Physics Volume 12, Seismology: Body Waves and Sources, pp. 231-266, ed. Bolt, B. A., Academic Press, New York.

Keller, J. B., 1962. Wave propagation in random media, Proc. Symp. appl. Math., 13, 227-246.

Madariaga, R., 1984. Gaussian beam synthetic seismograms in a vertically varying medium, Geophys. J.R. astr. Soc., 79, $589-612$.

Moore, B. J., 1980. Seismic ray theory for lithospheric structures with slight lateral variations, Geophys. J.R. astr. Soc., 63, 671-689.

Moore, B. J., 1989. Ray propagation through slightly heterogeneous media with application to the inversion of seismic data, Ultrasonics International 89 Conference Proceedings, pp. 1001-1006, Butterworths, Guildford, UK.

Newton, R. G., 1966. Scattering of Waves and Particles, McGraw-Hill, New York.

Nolet, G., 1987. Seismic wave propagation and seismic tomography, in Seismic Tomography with Applications in Global Seismology and Exploration Geophysics, pp. 1-23, ed. Nolet, G., Reidel, Dordrecht.

Sutton, G. R. \& Moore, B. J., 1987. Inversion of an unmigrated stacked section to determine an interval velocity model, Geophys. Prosp., 35, 895-907.

\section{APPENDIX A: SOLUTION TO EQUATION (18) IN PLANE GEOMETRY}

Under different conditions, equation (18) describes both the first-order corrections to ray geometry resulting from small fluctuations in the velocity field about a verically-varying model and the corrections to ray geometry resulting from small changes to the initial conditions when there are no fluctuations in the velocity field. Therefore $z$ is used rather than $x^{2}$ as the variable describing the correction to position on the ray and the equation is solved subject to non-zero initial conditions thus allowing the solution to both problems to be determined simultaneously. Specifically, our initial conditions will be

and

$$
\begin{aligned}
\mathbf{z}(0) & =\mathbf{q}(0) \\
\frac{d \mathbf{z}}{d s}(0) & =\delta(0) \text { with } \delta(0) \cdot \mathbf{p}^{0}(0)=0
\end{aligned}
$$

The condition on $\delta(0)$ ensures that $\frac{d \mathrm{x}}{d s}$ remains a unit vector to first order in small quantities.

From equation (18), it is seen that the 2-component of $\mathbf{2}$ satisfies:

$$
\begin{aligned}
& \frac{d}{d s}\left(u_{0} \frac{d z_{2}}{d s}\right)=u_{0} \nabla_{2}^{T} f_{1}\left(x_{0}\right)=u_{0} \nabla_{2} f_{1}\left(x_{0}\right) \\
& \text { and thus } \quad z_{2}(s)=\int_{0}^{s} B_{2}(s, \sigma) \nabla_{2} f_{1}\left(x^{0}(\sigma)\right) d \sigma+B_{2}(s, 0) \delta_{2}+q_{2} \\
& \text { where } \quad B_{2}(s, \sigma)=\int_{0} \frac{u_{0}(\sigma)}{u_{0}\left(s^{\prime \prime}\right)} d s^{\prime \prime}=\frac{u_{0}(\sigma)}{p}\left[x_{1}^{0}(s)-x_{1}^{0}(\sigma)\right]
\end{aligned}
$$

That is, $B_{2}(s, \sigma)$ is simply proportional to the horizontal displacement of the reference ray as the arclength parameter varies from $\sigma$ to $s$.

The equation for the 3-component of $\mathbf{z}$ is simplified by noting that at $\mu_{0}$ varies only with $x_{3}^{0}$.

$$
\frac{d u_{0}}{d s}=u_{0}^{\prime} \frac{d x_{3}^{0}}{d s}
$$

and so the basic equation for the 3-component of $z$ is:

$$
\frac{d^{2}}{d s^{2}}\left(u_{0} z_{3}\right)-z, u_{0}^{\prime \prime}=u_{0} \nabla_{3}^{T} f_{1}\left(x^{0}\right)
$$

Now

$$
\frac{d^{2} p_{3}^{0}}{d s^{2}}=\frac{d}{d s}\left(u_{0}^{0}\right)=u_{0}^{\prime \prime} \frac{d x_{3}^{0}}{d s}
$$


So provided $\frac{d x_{3}^{0}}{d s} \neq 0$, equation (A-4) may be multiplied throughout by $p_{3}^{0}$ to obtain:

$$
\frac{d}{d s}\left(p_{3}^{0} \frac{d}{d s}\left(u_{0} z_{3}\right)-u_{0} x_{3} \frac{d p_{3}^{0}}{d s}\right)=p_{3}^{0} u_{0} \nabla_{3}^{T} f_{1}\left(\mathbf{x}^{9}\right)
$$

$$
\begin{aligned}
& \text { Thus } \quad \frac{d}{d s}\left(\frac{\mu_{0} 2_{3}}{p_{3}^{0}}\right)=\frac{\phi_{3}(s)+c_{3}}{\left[p_{3}^{0}(s)\right]^{2}} \\
& \text { where } \quad \phi_{3}(s)=\int_{0}^{\prime} p_{3}^{0}(\sigma) u_{0}(\sigma) \nabla_{3}^{T} f_{1}\left(\mathbf{x}^{0}(\sigma) d \sigma\right. \\
& \text { and } \quad c_{3}=u_{0}(0)\left[p_{3}^{0}(0) \delta_{3}-p k(0) q_{3}\right] \\
& \text { with } \quad \kappa(s)=\left\|\frac{d^{2} x^{0}}{d s^{2}}\right\|=\frac{p u_{0}^{\prime}(s)}{u_{0}^{2}(s)} \text {, the curvature of the ray at } x^{0}(s) \text {. }
\end{aligned}
$$

$$
\begin{aligned}
& \text { It follows that } \quad z_{3}(s)=\int_{0}^{s} B_{3}^{*}(s, \sigma) \nabla_{3}^{T} f_{1}\left(\mathbf{x}^{0}(\sigma)\right) d \sigma+B_{3}^{*}(s, 0) \delta_{3}+C_{3}^{*}(s, 0) q_{3} \\
& \text { where } \quad B_{3}^{*}(s, \sigma)=\frac{u_{0}(\sigma)}{u_{0}(s)} \int_{\sigma}^{0} \frac{p_{3}^{0}(s) p_{3}^{0}(\sigma)}{\left[p_{3}^{0}\left(s^{\prime \prime}\right)\right]^{2}} d s^{\prime \prime} \\
& \text { and } \quad C_{3}^{*}(s, 0)=\frac{p_{3}^{0}(s) u_{0}(0)}{u_{0}(s) p_{3}^{0}(0)}-\frac{p \kappa(0)}{p_{3}^{0}(0)} B_{3}^{*}(s, 0) \\
& \text { The 1-component of } \mathbf{z} \text { satisfies } \\
& \frac{d}{d s}\left(u_{0} \frac{d z_{1}}{d s}\right)+\frac{d}{d s}\left(z_{3} \frac{d x_{1}^{0}}{d s} u_{0}^{\prime}\right)=u_{0} \nabla_{1}^{T} f_{1}\left(x^{0}\right) \\
& \text { where } \quad \frac{d x_{1}^{0}}{d s}=p \alpha_{0}(s)=\frac{p}{u_{0}(s)}
\end{aligned}
$$

Therefore integrating equation (A-9) and using initial conditions (A-1) to evaluate the constant of integration, we obtain

$$
\begin{aligned}
& \frac{d z_{1}}{d s}=\frac{1}{u_{0}(s)}\left\{\int_{0}^{a} u_{0}(\sigma) \nabla_{1}^{T} f_{1}\left(\mathbf{x}^{0}(\sigma)\right) d \sigma-\frac{z_{3}(s) p u_{0}^{\prime}(s)}{u_{0}(s)}+\frac{q_{3} p u_{0}^{\prime}(0)}{u_{0}(0)}+u_{0}(0) \delta_{1}\right\} \quad \text { and thus } \\
& z_{1}(s)=\int_{0}^{t} B_{2}(s, \sigma) \nabla_{1}^{T} f_{1}\left(x^{0}(\sigma)\right) d \sigma+p \int_{0}^{t} z_{3}(\sigma) \frac{d \alpha_{0}}{d x_{3}^{0}} d \sigma+u_{0}(0)\left\{x(0) q_{3}+\delta_{1}\right\} \int_{0}^{s} \frac{d s^{\prime \prime}}{u_{0}\left(s^{\prime \prime}\right)}+q_{1} \text { (A-10) }
\end{aligned}
$$

$$
\text { Now } \begin{aligned}
\frac{p_{3}^{0}(s)}{u_{0}(s)} & =\frac{d x_{3}^{0}}{d s} \text { and so from equation (A-7) we have } \\
z_{3}(s) \frac{d \alpha_{0}}{d x_{3}^{0}} & =\frac{d \alpha_{0}}{d s}\left\{\int_{0}^{2} \frac{\phi_{3}(\sigma)+c_{3}}{\left[p_{3}^{0}(\sigma)\right]^{2}} d \sigma+\frac{q_{3} u_{0}(0)}{p_{3}^{0}(0)}\right\}
\end{aligned}
$$

Therefore, we may integrate the second integral in equation (A-10) by parts to obtain

$$
p \int_{0}^{\star} z_{9}(\sigma) \frac{d \alpha_{0}}{d x_{3}^{0}} d \sigma=\int_{0}^{\star} F(s, \sigma) \nabla_{3}^{T} f_{1}\left(x^{0}(\sigma)\right) d \sigma+F(s, 0) \delta_{3}+G(s, 0) q_{3}
$$

$$
\begin{array}{ll}
\text { where } & \quad F(s, \sigma)=\int_{\sigma}^{s} \frac{u_{0}(\sigma) p_{3}^{0}(\sigma)}{\left[p_{3}^{0}\left(s^{\prime \prime}\right)\right]^{2}}\left[\frac{p}{u_{0}(s)}-\frac{p}{u_{0}\left(s^{\prime}\right)}\right] d s^{\prime \prime} \\
\text { and } & \quad G(s, 0)=\frac{p}{p_{3}^{0}(0)}\left\{\frac{u_{0}(0)}{u_{0}(s)}-[1+\kappa(0) F(s, 0)]\right\}
\end{array}
$$

The expressions for $F(s, \sigma)$ and $G(s, 0)$ vanish when $u_{0}\left(x_{j}^{0}\right)$ is constant at all depths. It is inappropriate to try and express $\nabla_{3}^{T} f_{1}\left(x^{0}(\sigma)\right)$ purely in terms of $\nabla_{1}^{I} f_{1}\left(x^{0}(\sigma)\right)$ as $\nabla_{1}^{T} f_{1}\left(x^{0}(\sigma)\right)$ vanishes at a turning point of the unperturbed ray whilst $\nabla_{y}^{\tau} f_{1}\left(\mathbf{x}^{0}(\sigma)\right)$ does not. Instead, it is preferable to express both the 1- and 3-components in terms of the in-plane component perpendicular to the ray (the n-component). The co-ordinate vectors are related as follows:

$$
\begin{array}{r}
\hat{A}=\frac{p}{u_{0}(s)} \hat{\mathbf{k}}-\frac{p_{3}^{0}(s)}{u_{0}(s)} \mathbf{i} \\
\mathbf{t}=\frac{d \mathbf{x}^{0}}{d s}=\frac{p_{3}^{0}(s)}{u_{0}(s)} \hat{\mathbf{k}}+\frac{p}{u_{0}(s)} \hat{\mathbf{i}}
\end{array}
$$

Now, from equation (11) we have

$$
\frac{d x^{0}}{d s} \cdot \nabla^{r} f_{1}\left(x^{0}\right)=0
$$

$$
\begin{aligned}
& \text { and so } \\
& \nabla_{3}^{T} f_{1}\left(x^{0}(\sigma)\right)=\frac{p}{u_{0}(\sigma)} \nabla^{\top} f_{1}\left(x^{0}(\sigma)\right) \\
& \nabla_{1}^{T} f_{1}\left(\mathbf{x}^{0}(\sigma)\right)=-\frac{p_{j}^{0}(\sigma)}{u_{0}(\sigma)} \nabla^{T} f_{1}\left(\mathbf{x}^{0}(\sigma)\right) \\
& \text { whilst } \\
& q_{3}=\frac{p_{3}^{0}(0) q_{1}+p q_{n}}{u_{0}(0)}, \quad q_{1}=\frac{p q_{1}-p_{3}^{0}(0) q_{n}}{u_{0}(0)} \\
& \delta_{3}=\frac{p \delta_{k}}{\mu_{0}(0)} \quad \text { and } \quad \delta_{1}=-\frac{p_{3}^{0}(0) \delta_{n}}{\mu_{0}(0)} \\
& \text { where } \quad B_{1}(s, \sigma)=\int_{\sigma}^{s} \frac{p_{3}^{0}(\sigma)}{\left[p_{3}^{0}\left(s^{\prime \prime}\right)\right]^{2}}\left[\frac{p^{2}}{u_{0}(s)}-u_{0}\left(s^{\prime \prime}\right)\right] d s^{\prime \prime} \\
& D_{1}(s, 0)=\frac{p}{u_{0}(s)}-\mathbf{k}(0) B_{1}(s, 0) \\
& C_{1}(s, 0)=-\frac{u_{0}(0)}{p_{3}^{0}(0)}+\frac{p}{p_{3}^{0}(0)} D_{1}(s, 0)
\end{aligned}
$$

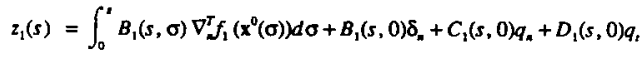

Similarly, we may write

$$
\begin{aligned}
z_{3}(s) & =\int_{0}^{s} B_{3}(s, \sigma) \nabla_{2}^{T} f_{1}\left(\mathbf{x}^{0}(\sigma)\right) d \sigma+B_{3}(s, 0) \delta_{a}+C_{3}(s, 0) q_{a}+D_{3}(s, 0) q_{t} \\
\text { where } \quad B_{3}(s, \sigma) & =\frac{p}{u_{0}(s)} \int_{\sigma}^{x} \frac{p_{3}^{0}(s) p_{3}^{0}(\sigma)}{\left[p_{3}^{0}\left(s^{\prime \prime}\right)\right]^{2}} d s^{\prime \prime} \\
D_{3}(s, 0) & =\frac{p_{3}^{0}(s)}{u_{0}(s)}-x(0) B_{3}(s, 0) \\
C_{3}(s, 0) & =\frac{p}{p_{3}^{0}(0)} D_{3}(s, 0)
\end{aligned}
$$

$$
\begin{aligned}
& \text { It follows that the solution to equation (18) in plane geometry may be written: } \\
& z(s)=\int_{0}^{4} P_{2}(s, \sigma) \nabla^{T} f_{1}\left(x^{0}(\sigma)\right) d \sigma+P_{2}(s, 0) \delta+P_{1}(s, 0) q \\
& z(s)=z_{n}(s) \hat{n}(s)+z_{2}(s) \hat{j}+z_{1}(s) t(s) \\
& \delta(s)=\delta_{n}(s) \hat{\mathrm{n}}(s)+\delta_{2}(s) \hat{\mathrm{j}} \\
& \mathbf{P}_{1}=\left(\begin{array}{lll}
R_{1} & 0 & Q_{1} \\
0 & R_{2} & 0 \\
R_{3} & 0 & Q_{3}
\end{array}\right), \quad \mathbf{P}_{2}=\left(\begin{array}{ll}
S_{1} & 0 \\
0 & S_{2} \\
S_{3} & 0
\end{array}\right) \\
& \text { with } \quad s_{1}(s, \sigma)=\frac{1}{u_{0}(s)} \int_{\sigma}^{\rho} \frac{p_{3}^{0}(s) p_{3}^{0}(\sigma) u_{0}\left(s^{\prime \prime}\right)}{\left[p_{3}^{0}\left(s^{\prime \prime}\right)\right]^{2}} d s^{\prime \prime} \\
& S_{2}(s, \sigma)=B_{2}(s, \sigma) \\
& s_{3}(s, \sigma)=\int_{0}^{s} \frac{p p_{3}^{0}(\sigma)}{\left[p_{3}^{0}\left(s^{\prime \prime}\right)\right]^{2}}\left[1-\frac{u_{0}\left(s^{\prime \prime}\right)}{u_{0}(s)}\right] d s^{\prime \prime} \\
& R_{1}(s, 0)=\frac{p_{3}^{0}(s) u_{0}(0)}{p_{3}^{0}(0) u_{0}(s)}-\frac{p \kappa(0)}{p_{3}^{0}(0)} S_{1}(s, 0) \\
& R_{2}(s, 0)=1 \\
& R_{3}(s, 0)=\frac{p}{p_{3}^{0}(0)}\left[1-\frac{u_{0}(0)}{u_{0}(s)}-\kappa(0) S_{3}(s, 0)\right] \\
& Q_{1}(s, 0)=-\kappa(0) S_{1}(s, 0) \\
& Q_{3}(s, 0)=1-x(0) S_{3}(s, 0) \text {. }
\end{aligned}
$$

From these expressions, it is clear that the terms $S_{3}(s, \sigma)$ and $R_{3}(s, 0)$ may only be neglected when $u_{0}(\mathbf{x})$ does not vary greatly along the raypath; otherwise the component of $\mathbf{z}$ along the ray is significant. The $Q$-factors describe the effect of a small initial displacement in the direction of the ray and give insight into the curvature terms in the $R$-factors.

An expression for $\frac{d z}{d s}$ may be obtained by differentiating the expressions for $z_{1}, z_{2}$ and $z_{3}$ with respect to $s$ :

$$
\frac{d \mathrm{z}}{d s}=\int_{0}^{x} \mathbf{P}_{4}(s, \sigma) \nabla^{r} f_{1}\left(\mathbf{x}^{0}(\sigma)\right) d \sigma+\mathbf{P}_{4}(s, 0) \delta+\mathbf{P}_{3}(s, 0) \mathbf{q}
$$


where

$$
\begin{aligned}
& P_{3}=\left(\begin{array}{ccc}
R_{4} & 0 & Q_{4} \\
0 & R_{5} & 0
\end{array}\right), \quad P_{4}=\left(\begin{array}{cc}
S_{4} & 0 \\
0 & S_{5}
\end{array}\right) \\
& s_{d}(s, \sigma)=\frac{p_{3}^{0}(\sigma)}{p_{3}^{0}(s)}+\frac{p \kappa(s)}{p_{3}^{0}(s)} \int_{\sigma}^{0} \frac{p_{3}^{0}(s) p_{3}^{0}(\sigma)}{\left[p_{3}^{0}\left(s^{\prime}\right)\right]^{2}} d s^{\prime \prime} \\
& S,(s, \sigma)=\frac{\mu_{0}(\sigma)}{\mu_{0}(s)} \\
& R_{4}(s, 0)=\frac{p}{p_{3}^{0}(0)}\left[\kappa(s)-\kappa(0) S_{4}(s, 0)\right] \\
& R_{s}(s, 0)=0 \\
& Q_{4}(s, 0)=x(s)-x(0) S_{4}(s, 0)
\end{aligned}
$$

It is easy to verify that the tangential component of $\frac{d z}{d s}$ is zero as may be proved directly from equation (18) by taking its scalar product with $\frac{d \mathrm{x}^{0}}{d s}$ and using equation (A-13) together with the fact that $\frac{d}{d s}\left(\frac{d \mathrm{x}^{0}}{d s}\right)$ is perpendicular to $\frac{d \mathrm{x}^{0}}{d s}$. This gives

$$
\frac{d}{d s}\left(u_{0} \frac{d \mathbf{x}^{0}}{d s} \cdot \frac{d \mathbf{z}}{d s}\right)-u_{0} \frac{d^{2} \mathbf{x}^{0}}{d s^{2}} \cdot \frac{d \mathbf{z}}{d s}+\frac{d}{d s}\left(\mathbf{z} \cdot \nabla u_{0}\right)-\frac{d x_{k}^{0}}{d s} z_{0} u_{0, i}=0
$$

Equation (A-16) may be simplified using equations (7) and (A-5) together with the fact that $u_{0}$ varies only with depth to give:

$$
\frac{d}{d s}\left(u_{0} \frac{d \mathrm{x}^{0}}{d s} \cdot \frac{d \mathrm{z}}{d s}\right)+\left(\frac{d \mathrm{x}^{0}}{d s} \cdot \nabla u_{0}\right) \frac{d \mathrm{x}^{0}}{d s} \cdot \frac{d \mathrm{z}}{d s}=0
$$

$$
\begin{aligned}
& \text { That is, } \quad \frac{1}{u_{0}} \frac{d}{d s}\left\{u_{0}^{2} \frac{d \mathrm{x}^{0}}{d s} \cdot \frac{d \mathrm{z}}{d s}\right\}=0 \\
& \text { with solution } \\
& \frac{d \mathrm{x}^{0}}{d s} \cdot \frac{d \mathrm{z}}{d s}=\frac{A}{u_{0}^{2}(s)}
\end{aligned}
$$

Now at $s=0$, the initial conditions required $A=0$. Thus at all points along the ray, $\frac{d \mathrm{z}}{d s}$ is perpendicular to the direction of the unperturbed ray. Equation (A-17) may be further integrated to obtain the expression for the tangential component of 2 given at equation (A-14).

\section{APPENDIX B: SOME PROPERTIES OF THE SPHERICAL POLAR COORDINATE SYSTEM}

Spherical co-ordinates are related to cartesian co-ordinates of position by the following relations:

$$
x=r \sin \theta \cos \phi, \quad y=r \sin \theta \sin \phi, \quad z=r \cos \theta
$$

Consequenty, the associated unit vectors and scale factors are:

$$
\begin{aligned}
& \boldsymbol{t}=\sin \theta \cos \phi \mathbf{i}+\sin \theta \sin \phi \mathbf{j}+\cos \theta \mathbf{k}, \quad h_{r}=1 \\
& \theta=\cos \theta \cos \phi i+\cos \theta \sin \phi \mathbf{j}-\sin \theta \mathbf{k}, \quad h_{0}=r \\
& \bar{\phi}=-\sin \phi 1+\cos \phi \mathrm{J}, \quad h_{\phi}=r \sin \theta
\end{aligned}
$$

These unit vectors vary with position and have partial derivatives:

$$
\begin{array}{lll}
\frac{\partial \hat{r}}{\partial r}=0, & \frac{\partial t}{\partial \theta}=\theta, & \frac{\partial \hat{r}}{\partial \phi}=\sin \theta \hat{\phi} \\
\frac{\partial \theta}{\partial r}=0, & \frac{\partial \theta}{\partial \theta}=-\hat{r}, & \frac{\partial \theta}{\partial \phi}=\cos \theta \hat{\phi} \\
\frac{\partial \hat{\phi}}{\partial \phi}=0, & \frac{\partial \hat{\phi}}{\partial \phi}=0, & \frac{\partial \hat{\phi}}{\partial \theta}=-(\sin \theta \hat{r}+\cos \theta \hat{\theta})
\end{array}
$$

In the plane of the unperturbed ray, $\phi$ remains constant and only $r$ and $\theta$ vary with position on the ray. Consequently.

$$
\frac{d t}{d s}=\theta \theta, \frac{d \theta}{d s}=-\theta \hat{r}, \frac{d \hat{\phi}}{d s}=0
$$

where denotes differentiation with respect to arclength along the ray.

\section{APPENDIX C: SOLUTION OF EQUATION (18) IN SPHERICAL GEOMETRY}

As in Appendix A, $\mathbf{z}$ is used rather than $x^{2}$ as the variable describing the correction to position on the ray and our initial conditions are:

$$
\begin{aligned}
& \text { and } \begin{array}{l}
\mathrm{z}(0)=\mathrm{q}(0) \\
\frac{d \mathrm{z}}{d s}(0)=\delta(0) \text { with } \delta(0) \cdot e=0
\end{array} \\
& \text { The condition on } \delta(0) \text { ensures that } \frac{d \mathrm{x}}{d s} \text { remain a unit vector to first order small quantities. }
\end{aligned}
$$

First of all, we consider the in-plane components of the solution. Taking the scalar product of equation (18) with $\frac{d \mathrm{x}^{0}}{d s}$ and using the fact that $\frac{d \mathrm{x}^{0}}{d s} \cdot \frac{d^{2} \mathrm{x}^{0}}{d s^{2}}=0$ gives:

$$
\frac{d}{d s}\left(u_{0} \frac{d \mathbf{x}^{0}}{d s} \cdot \frac{d \mathbf{z}}{d s}\right)-u_{0} \frac{d \mathbf{z}}{d s} \cdot \frac{d^{2} \mathbf{x}^{0}}{d s^{2}}+\frac{d}{d s}\left\{z, u_{0}^{\prime}\right\rangle-\left(\hat{r} \cdot \frac{d \mathbf{x}^{0}}{d s}\right) z, u_{0}^{\prime \prime}-\left(\frac{\hat{\theta}}{r_{0}} \cdot \frac{d \mathbf{x}^{0}}{d s}\right) z_{0} \mu_{0}^{\prime}=0
$$

where $z_{r}=\mathbf{z} \cdot \hat{\mathbf{r}}$ and $z_{\theta}=\mathbf{z} \cdot \hat{\theta}$.

Equations (7), (14) and (15) may be used to simplify the equation to obtain:

$$
\frac{d}{d s}\left(u_{0} \frac{d \mathbf{x}^{0}}{d s} \cdot \frac{d \mathrm{z}}{d s}\right)-u_{0}^{\prime}\left(\vec{r} \cdot \frac{d \mathrm{z}}{d s}\right)+\dot{u}_{0} \frac{d \mathbf{x}^{0}}{d s} \cdot \frac{d \mathrm{z}}{d s}+\frac{d}{d s}\left(u_{0}^{\prime} z_{,}\right)-z_{,} u_{0}{ }^{\prime \prime} \dot{r}_{0}-z_{0} \mu_{0}^{\prime} \theta=0
$$

Furthermore we have:

$$
\frac{d \mathbf{z}}{d s}=\left(\dot{z},-z_{\theta} \dot{\theta}\right) \hat{r}(s)+\left(z_{\theta}+z_{r} \dot{\theta}\right) \ddot{\theta}(s)+z_{\theta} \bar{\phi}(s)
$$

whilst

$$
\frac{d}{d s}\left(u_{0}^{\prime} z_{r}\right)-z_{r} u_{0}^{\prime \prime} r_{0}=u_{0}^{\prime} z_{r}
$$

Substituting in equation (C-2) gives:

$$
\frac{d}{d s}\left(u_{0} \frac{d \mathbf{x}^{0}}{d s} \cdot \frac{d x}{d s}\right)+\dot{u}_{0} \frac{d \mathbf{x}^{0}}{d s} \cdot \frac{d \mathbf{z}}{d s}=0
$$

It follows that

$$
\frac{d \mathrm{x}^{0}}{d s} \cdot \frac{d \mathrm{z}}{d s}=\frac{A}{u_{0}^{2}(x)}
$$

For the initial conditions given both in equation (19) and in equation (C-1), we obtain $A=0$ as before and we find that at all points along the ray, $\frac{d z}{d s}$ is perpendicular to the direction of the unperturbed ray, that is, it lies in the wavefront at each point.

Next we take the scalar product of equation (18) with $x^{0}=r_{0} t^{2}$ to obtain:

$$
\frac{d}{d s}\left(u_{0} \frac{d \mathrm{z}}{d s} \cdot \mathrm{x}^{0}+u_{0} z \cdot x^{0}\right)-z, u_{0}^{\prime}-r_{0} z, u_{0}^{\prime \prime}=u_{0} x^{0} \cdot \nabla^{\mathrm{r}} f_{1}\left(\mathrm{x}^{0}\right)
$$

Now, from equation (7) and (C-4) we have

$$
\frac{d}{d s}\left(u_{0} \frac{d \mathbf{x}^{0}}{d s} \cdot \mathbf{z}\right)=\mathbf{z} \cdot \frac{d}{d s}\left(u_{0} \frac{d \mathbf{x}^{0}}{d s}\right)=u_{0}^{0} z
$$

and so equation (C-5) may be written

$$
\frac{d^{2}}{d s^{2}}\left(u_{0} r_{0} z_{r}\right)-u_{0} r_{0} z_{r}\left(\frac{2 u_{0}^{\prime}}{r_{0} u_{0}}+\frac{u_{0}^{\prime \prime}}{u_{0}}\right)=u_{0} x^{0} \cdot \nabla^{T} f_{1}\left(x^{q}\right)
$$

$$
\text { Noting that } \quad \frac{2 u_{0}^{\prime}}{r_{0} u_{0}}+\frac{u_{0}^{\prime \prime}}{u_{0}}=\frac{\left(r_{0} u_{0}\right)^{\prime \prime}}{r_{0} u_{0}}
$$

it is seen that equation (C-7) does, in fact, have essentially the same form as equation (A-6) and the solution proceeds in a similar way. Provided that $t_{0} \neq 0$, it is possible to multiply equation (C-7) 


$$
\begin{aligned}
& \text { throughout by } p_{j}^{0}=u_{\sigma} \sigma_{0} t_{0}=\sqrt{\left(u_{0} r_{0}\right)^{2}-p^{2}} \text { which gives: } \\
& z,(s)=\frac{p_{3}^{0}(s)}{u_{0}(s) r_{0}(s)}\left\{\int_{0}^{1} C_{3}^{0}(s, \sigma) \nabla_{1}^{t} f_{1}\left(x^{0}(\sigma)\right) d \sigma+C_{3}^{*}(s, 0) \delta_{,}+D_{3}^{j}(s, 0) q\right\} \\
& \text { where } \quad C_{3}^{*}(s, \sigma)=\int_{0}^{0} \frac{p_{3}^{0}(\sigma) u_{0}(\sigma) r_{0}(\sigma)}{\left[p_{3}^{0}\left(s^{\prime}\right)\right]^{2}} \\
& D_{3}^{*}(s, 0)=\frac{u_{0}(0) y_{0}}{p_{3}^{0}(0)}-\frac{p L(0)}{p_{3}^{0}(0)} C_{3}^{*}(s, 0) \\
& \nabla_{,}^{\tau} f_{1}=p \cdot \nabla^{T} f_{1} \\
& L(0)=\frac{p\left(u_{0} \gamma_{0}\right)^{\prime}}{u_{0}^{2} r_{0}^{2}} l_{,-0}=\frac{p}{y_{0}^{2} u_{0}(0)}+x(0)
\end{aligned}
$$

and $k(0)$ is the curvature of the ray at $x^{0}(0)$ as defined at equation (C-14).

Equarion (C-8) may be used in conjunction with equation (C-4) to derive an expression for the component of $\mathrm{z}$ direcied along the nay:

$$
\begin{aligned}
& 2 \cdot \frac{d \mathrm{x}^{0}}{d s}=\int_{0}^{0} C_{3}(s, \sigma) \nabla_{s}^{\tau} f_{1}\left(\mathrm{x}^{0}(\sigma)\right) d \sigma+C_{s}(s, 0) \delta_{2}+D_{3}(s, 0) q_{1}+\frac{u_{0}(0)}{u_{0}(s)} q_{t} \\
& \text { where } \quad C_{s}(s, \sigma)=\int_{0}^{s} \frac{r_{0}(\sigma) u_{0}(\sigma) p_{j}^{0}(\sigma)}{\left[p_{j}^{0}\left(s^{\prime \prime}\right)\right]^{2}}\left[1-\frac{\mu_{0}\left(s^{\prime \prime}\right)}{\mu_{0}(s)}\right] d s^{\prime \prime} \\
& \text { and } \quad D_{s}(s, 0)=\left[1-\frac{u_{0}(0)}{u_{0}(s)}\right] \frac{u_{0}(0) y_{0}}{p_{3}^{0}(0)}-\frac{p L(0)}{p_{3}^{0}(0)} C_{5}(s, 0)
\end{aligned}
$$

At first sight, it may seem unusual to have $\frac{u_{0}\left(s^{\prime \prime}\right)}{u_{0}(s)}$ rather than $\frac{r_{0}\left(s^{\prime}\right) u_{0}\left(s^{\prime \prime}\right)}{r_{0}(s) u_{0}(s)}$ in a problem involving spherical symmery. However, in the special case $\mu_{0}\left(r_{0}\right)=$ constant at all depths, we see that this particular formulation does indeed cause the integral for $C$, to vanish and thus gives an $\mathrm{x}^{2}(s)$ which lies in the wavefront of the zeroth-order disturbance at $\mathbf{x}^{0}(s)$, as found in paper 1 .

Equarion (C-9) may be used to derive an expression for $z_{0}$. From equations (14) and (15) we have:

$$
\begin{aligned}
& \mathbf{z} \cdot \frac{d \mathbf{x}^{0}}{d s}=\frac{\left(p z_{0}+p_{1}^{0} z_{0}\right)}{r_{0} u_{0}} \\
& \text { and so } \quad z_{0}(s)=\frac{-p_{1}^{0}(s)}{p} z_{r}(s)+\frac{r_{0}(s) u_{0}(s)}{p}\left(\mathbf{z} \cdot \frac{d \mathbf{x}^{0}}{d s}\right)
\end{aligned}
$$

Once again, it is more appropriate to express $r$ - and $\theta$-components in terms of a component along the ray (the $t$-component) and an in-plane component perpendicular to the ray (the $n$-component). The co-ordinate vectors are related as follows:

$$
\begin{aligned}
& \hat{\mathbf{n}}(s)=\frac{p}{r_{0}(s) u_{0}(s)} \hat{P}(s)-\frac{p_{j}^{0}(s)}{r_{0}(s) u_{0}(s)} \hat{\theta}(s) \\
& \text { and } \quad t(s)=\frac{d \mathrm{x}^{0}}{d s}=\frac{p_{j}^{0}(s)}{r_{0}(s) \mu_{0}(s)} \mathrm{r}(s)+\frac{p}{r_{0}(s) u_{0}(s)} \theta(s) \\
& \text { Then } \quad q_{1}=\frac{p q_{n}+p_{3}^{0}(0) q_{1}}{r_{0}(0) u_{0}(0)}, q_{0}=\frac{p q_{1}-p_{j}^{0}(0) q_{n}}{r_{0}(0) u_{0}(0)} \\
& \delta_{r}=\frac{p}{r_{0}(0) u_{0}(0)} \delta_{n} \text { and } \delta_{0}=\frac{-p_{3}^{0}(0)}{r_{0}(0) u_{0}(0)} \delta_{n} \text {. }
\end{aligned}
$$

$$
\text { Thus we may write }
$$$$
z_{n}(s)=\int_{0}^{s} S_{1}(s, \sigma) \nabla_{J}^{T} f_{1}\left(\mathbf{x}^{0}(\sigma)\right) d \sigma+S_{1}(s, 0) \delta_{n}+R_{1}(s, 0) q_{n}+Q_{1}(s, 0) q_{1}
$$$$
\text { where } \quad s_{1}(s, \sigma)=\frac{1}{u_{0}(s)} \int_{0}^{0} \frac{p_{j}^{0}(s) p_{1}^{0}(\sigma) u_{0}\left(s^{\prime \prime}\right)}{\left[p_{j}^{0}\left(s^{\prime \prime}\right)\right]^{2}} d s^{\prime \prime}
$$$$
R_{1}(s, 0)=\frac{p_{3}^{0}(s) \mu_{0}(0)}{p_{3}^{0}(0) L_{0}(s)}-\frac{p L(0)}{p_{3}^{0}(0)} S_{1}(s, 0)
$$$$
Q_{1}(s, 0)=-L(0) S_{1}(s, 0)
$$

and

$$
z_{1}(s)=\int_{0}^{a} S_{3}(s, \sigma) \nabla_{J_{1}}^{r}\left(\mathbf{x}^{0}(\sigma)\right) d \sigma+S_{3}(s, 0) \delta_{n}+R_{3}(s, 0) q_{n}+Q_{3}(s, 0) q_{1}
$$

$$
\text { where } \begin{aligned}
S_{3}(s, \sigma) & =\int_{0}^{c} \frac{p p_{3}^{0}(\sigma)}{\left[p_{3}^{0}\left(s^{\prime \prime}\right)\right]^{2}}\left[1-\frac{u_{0}\left(s^{\prime \prime}\right)}{u_{0}(s)}\right] d s^{\prime \prime} \\
R_{3}(s, 0) & =\frac{p}{p_{3}^{0}(0)}\left[1-\frac{u_{0}(0)}{u_{0}(s)}\right]-\frac{p L(0)}{p_{3}^{0}(0)} S_{3}(s, 0) \\
Q_{3}(s, 0) & =1-L(0) S_{3}(s, 0)
\end{aligned}
$$

Finally, from equations (18) and (B-4) it is seen that the $\phi$-component of $\mathbf{z}$ satisfies the equation:

$$
r_{0} \frac{d}{d s}\left(u_{0} \frac{d z_{0}}{d s}\right)-z_{0} \frac{d u_{0}}{d r_{0}}=r_{0} u_{0} \nabla_{\Psi_{1}}^{T}\left(\mathrm{x}^{0}\right)
$$

Multiplying through the unit vector by $f$ and using equation (6) gives:

$$
\frac{d}{d s}\left(\mathrm{x}^{0} u_{0} \frac{d z_{0}}{d s}-z_{0} u_{0} \frac{d \mathbf{x}^{0}}{d s}\right)=\mathbf{x}^{0} u_{0} \nabla^{T} f_{1}\left(\mathbf{x}^{0}\right)
$$

Integrating with respect to $s$ and taking the $\delta(s)$ component of equation (C-12), using the orhogonality of the unit vectors $\hat{r}(s)$ and $\theta(s)$ gives:

$$
\begin{aligned}
z_{0}(s) & =\int_{0}^{1} S_{2}(s, \sigma) \nabla^{r} f_{1}\left(x^{0}(\sigma)\right) d \sigma+S_{2}(s, 0) \delta_{0}+R_{2}(s, 0) q_{4} \\
\text { where } & S_{2}(s, \sigma)=-\frac{r_{0}(\sigma) u_{0}(\sigma)}{p} r_{0}(s) \dot{\theta}(s) \cdot \dot{r}(\sigma) \\
\text { and } & R_{2}(s, 0)=\frac{u_{0}(0) r_{0}(s)}{p} \dot{\theta}(s) \cdot \mathrm{e}
\end{aligned}
$$

Thus we may write the solution to equation (18) in spherical geometry as:

$$
\begin{gathered}
\mathbf{z}(s)=\int_{0}^{\prime} \mathbf{P}_{2}(s, \sigma) \nabla^{T} f_{1}\left(\mathbf{x}^{0}(\sigma)\right) d \sigma+\mathbf{P}_{2}(s, 0) \delta+\mathbf{P}_{1}(s, 0) \mathbf{q} \\
\text { where } \\
\mathbf{P}_{1}=\left(\begin{array}{lll}
R_{1} & 0 & Q_{1} \\
0 & R_{2} & 0 \\
R_{1} & 0 & Q_{3}
\end{array}\right) \text { and } \mathbf{P}_{2}=\left(\begin{array}{ll}
S_{1} & 0 \\
0 & S_{2} \\
S_{3} & 0
\end{array}\right)
\end{gathered}
$$

and the 1-, 2- and 3-components refer to components in the A-, $\hat{\phi}$ - and $\mathrm{t}$-directions respectively. As in Appendix $A$, the components in the direction of the ray are only negligible when $u_{0}(x)$ does not vary greatly along the raypath.

This ray co-ordinate system is particularly convenient to use if expressions for $\frac{d z}{d s}$ are required. We note that

$$
\frac{d \hat{A}}{d s}=-\kappa(s) t(s) \text { and } \frac{d t}{d s}=K(s) \hat{n}(s)
$$

where $\quad k(s)=\frac{p \frac{s}{s_{0}}(s)}{r_{0}(s) \mu_{0}^{2}(s)}$

Therefore

$$
\frac{d z}{d s}=\left(t_{f}(s)-\kappa(s) z_{n}(s)\right) t(s)+\left(z_{n}(s)+K(s) z_{1}(s)\right) \hat{n}(s)+z_{q}(s) \hat{\phi}(s)
$$

and it is easily verified that $z_{1}(s)=x(s) z_{n}(s)$ as required by equation (C-4).

$$
\begin{aligned}
& \text { Thus } \quad \frac{d z}{d s}=\int_{0}^{s} P_{d}(s, \sigma) \nabla^{T} f_{1}\left(x^{0}(\sigma)\right) d \sigma+P_{d}(s, 0) \delta+P_{3}(s, 0) q \\
& \text { where } \quad \mathbf{P}_{3}=\left(\begin{array}{lll}
R_{4} & 0 & Q_{4} \\
0 & R_{1} & 0
\end{array}\right) \text { and } \mathbf{P}_{4}=\left(\begin{array}{ll}
S_{4} & 0 \\
0 & S_{5}
\end{array}\right) \\
& \text { with } \quad S_{4}(s, \sigma)=\frac{p_{3}^{0}(\sigma)}{p_{3}^{0}(s)}+p_{3}^{0}(\sigma) \int_{\sigma}^{\prime} \frac{p \mathbf{K}(s)+u_{0}\left(s^{\prime \prime}\right)}{\left[p_{3}^{0}\left(s^{\prime \prime}\right)\right]^{2}} d s^{\prime \prime} \\
& S_{5}(s, \sigma)=\frac{r_{0}(\sigma) u_{0}(\sigma)}{p} \hat{n}(s) \cdot \hat{r}(\sigma) \\
& R_{4}(s, \sigma)=\frac{u_{0}(0)+p K(s)}{p_{3}^{0}(0)}-\frac{p L(0)}{p_{3}^{0}(0)} S_{4}(s, 0) \\
& R_{5}(s, \sigma)=-\frac{\mu_{0}(0)}{p} \mathrm{~h}(s) \cdot \mathrm{e} \\
& Q_{4}(s, \sigma)=x(s)-L(0) S_{4}(s, 0) \text {. }
\end{aligned}
$$




\section{APPENDIX D: SOME PROPERTIES OF THE CURVILINEAR RAY GEOMETRY}

We choose a set of orthogonal curvilinear ray co-ordinates $(\tau, \xi, \eta)$ where $\tau$ denotes travel-ime along the ray and $\xi, \eta$ denote any orthogonal co-ordinates on the wavefront. These ray co-ordinates have associated with them unit vectors and scale factors defined as follows:

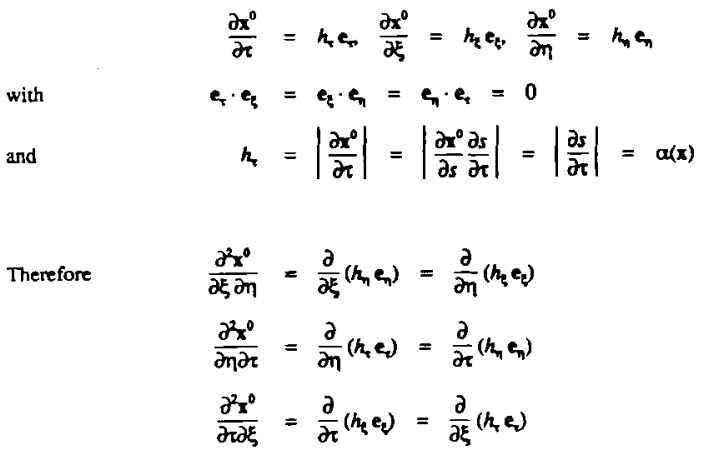

Thus, $\quad \frac{\partial h_{\eta}}{\partial \xi} \epsilon_{n}+h_{n} \frac{\partial e_{n}}{\partial \xi}=\frac{\partial h_{\xi}}{\partial \eta} e_{\xi}+h_{3} \frac{\partial e_{\xi}}{\partial \eta}$

$$
\begin{aligned}
& \frac{\partial h_{\tau}}{\partial \eta} e_{i}+h_{i}+\frac{\partial e_{r}}{\partial \eta}=\frac{\partial h_{m}}{\partial \tau} e_{i}+h_{\eta} \frac{\partial e_{\eta}}{\partial \tau} \\
& \frac{\partial h_{\xi}}{\partial \tau} e_{\xi}+h_{\xi} \frac{\partial e_{\xi}}{\partial \tau}=\frac{\partial h_{\tau}}{\partial \xi} e_{z}+h_{i} \frac{\partial e_{s}}{\partial \xi}
\end{aligned}
$$

Now the onhogonality of the unit vectors may be used in equations (D-3) to obtain:

$$
\begin{aligned}
& \frac{\partial h_{m}}{\partial \xi}=h_{q} e_{n} \cdot \frac{\partial e_{\xi}}{\partial m}=-h_{\xi} e_{q} \cdot \frac{\partial e_{n}}{\partial m} \\
& \frac{\partial h_{\xi}}{\partial \eta}=h_{n} e_{\xi} \cdot \frac{\partial e_{n}}{\partial \xi}=-h_{n} e_{n} \cdot \frac{\partial e_{\xi}}{\partial \xi}
\end{aligned}
$$

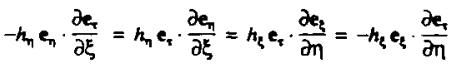

and similar relations from the remaining two equations in (D-3). In fact, it may be deduced from the full set of relations that each of the four expressions in equations (D-4c) is zero and similarly for cyclic permutation of the co-ordinate symbols.

So far, we have made no assumptions about the geometry of the co-ordinate system beyond the orthogonality of the co-ordinate vectors and so the results are easily adapted to the situation when arclength $s$ is the co-ordinate along the ray simply by replacing $h_{r}$ by $h$, in the previous equations. It is interesting to see the implications of equations (D-4) when we choose $\eta$ as the $n$ co-ordinate defined in section 2 and $\xi$ as either the 2-component (for plane geomery) or the component (for spherical geometry). Then we have:

$$
\frac{\partial e_{k}}{\partial s}=-\kappa(s) e_{k}, \frac{\partial e_{A}}{\partial s}=\kappa(s) e_{4}, \frac{\partial e_{\xi}}{\partial s}=0
$$

where

$$
\kappa(s)=\left|\nabla^{r} \ln \mu_{0}\right| \quad \text { the curvature of the ray at } x^{o}(s) \text {. }
$$

Using these three results in the relations obtained from equation (D-4) by cyclic permutation of the co-ordinate symbols, we find that:

$$
\frac{\partial h_{s}}{\partial \eta}=h_{n} \boldsymbol{k}(s), \frac{\partial h_{s}}{\partial \xi}=0
$$

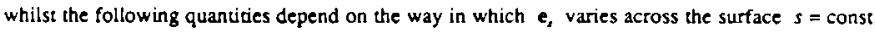
that is, in a direction transverse to the ray:

$$
\begin{aligned}
& \frac{\partial h_{n}}{\partial s}=h_{1} e_{n} \cdot \frac{\partial e_{i}}{\partial \eta}=-h_{,}, \cdot \frac{\partial e_{n}}{\partial \eta} \\
& \frac{\partial h_{\xi}}{\partial s}=h_{1} e_{\xi} \cdot \frac{\partial e_{s}}{\partial \xi}=-h_{i} e_{1} \cdot \frac{\partial e_{\xi}}{\partial \xi}
\end{aligned}
$$

However, in the special case of ray-centred co-ordinates, each term in equations (D-7) is zero.

The quancities in the first two equations of (D-4) are themselves unconstrained by equations (D-5). So care is needed in handling the derivatives of the scale factors in applications.

One function of the ray geometry which appears in the small perturbation analysis is the vector quantity $\gamma$ given by:

$$
\gamma=\frac{1}{h_{1} h_{q} h_{\eta}}\left(\frac{\partial}{\partial \xi}\left(h_{h_{n}} h_{\xi}\right)+\frac{\partial}{\partial \eta}\left(h_{h} h_{\xi} e_{\eta}\right)\right)
$$

Equations (D-4) and similar equations obtained by cyclic permutation of the co-ondinate symbols may be used to simplify the expression to obtain:

$$
\begin{aligned}
\gamma & =\nabla^{r} \ln u+\frac{1}{h_{\tau}} \frac{\partial \ln J}{\partial \tau} \mathrm{e}_{\tau} \\
\text { where } \quad J & =h_{\xi} h_{\pi}=\left|\frac{\partial \mathbf{x}^{0}}{\partial \xi} \times \frac{\partial x^{0}}{\partial \eta}\right|,
\end{aligned}
$$

the area of surface element on the wavefront.

Another function of the ray geometry which is used in this paper is $\nabla\left(\frac{d \mathrm{x}^{0}}{d s}\right)$ which is related to

the phase curvature function $A_{4}=\tau_{i}$ as follows:

$$
A_{\star}=\left(u \frac{d x_{i}^{0}}{d s}\right)_{*}=u_{* k} \frac{d x_{i}^{0}}{d s}+u\left(\frac{d x_{i}^{0}}{d s}\right),
$$

Now

$$
\begin{aligned}
& \frac{\partial}{\partial \xi}\left(u e_{q}\right)=\frac{\partial u}{\partial \xi} e_{q}+u \frac{\partial e_{\tau}}{\partial \xi}=\frac{\partial u}{\partial \xi} e_{q}+\frac{u}{h_{q}} \frac{\partial h_{\xi}}{\partial \tau} e_{\xi} \\
& \frac{\partial}{\partial \eta}\left(u e_{\tau}\right)=\frac{\partial u}{\partial \eta} e_{\tau}+u \frac{\partial e_{\tau}}{\partial \eta}=\frac{\partial u}{\partial \eta} e_{\tau}+\frac{u}{h_{\tau}} \frac{\partial h_{\eta}}{\partial \tau} e_{\eta}
\end{aligned}
$$

and, from the ray equation we have:

$$
\frac{\partial}{\partial \tau}\left(u e_{\tau}\right)=\frac{\partial s}{\partial \tau} \frac{\partial}{\partial s}\left(u e_{s}\right)=h_{T} \nabla u
$$

Thus, the expression for the phase curvature function is given by:

$A_{i k}=\frac{1}{h_{\tau}}\left[\frac{\partial u}{\partial \tau} e_{\tau} e_{\tau}+u \frac{\partial \ln h_{\xi}}{\partial \tau} e_{q} e_{q}+u \frac{\partial \ln h_{\eta}}{\partial \tau} e_{\eta} e_{m}\right]+\frac{1}{h_{l}} \frac{\partial u}{\partial \xi}\left[e_{\xi} e_{i}+e_{\tau} e_{\xi}\right]+\frac{1}{h_{\eta}} \frac{\partial u}{\partial \eta}\left[e_{\eta} e_{\tau}+e_{q} e_{\eta}\right]$ (D-13) where the suffix $i$ refers to the second term of each dyad and the suffix $k$ refers to the first term in each case. Equation (D-13) shows the symmetry of the dyadic $A_{\star}$. The expression for $\mu \nabla\left(\frac{d x^{0}}{d s}\right)$ differs from (D-13) by the term $\nabla_{u}$, which removes the symmerry from the dyadic; it also removes all terms from the dyadic with $i$ component in the $e_{\text {q }}$ direction, a significant result:

$$
u \nabla\left(\frac{d \mathbf{x}^{0}}{d s}\right)=\frac{u}{h_{i}}\left[\frac{\partial \ln h_{\xi}}{\partial \tau} e_{\xi} e_{\xi}+\frac{\partial \ln h_{\eta}}{\partial t} e_{\eta} e_{\eta}\right]+e_{\tau} \nabla^{T} u
$$

In ray-centred co-ordinates, this reduced further to:

$$
\nabla\left(\frac{d x^{0}}{d s}\right)=e_{r} \nabla^{r} \ln u=x(s) e_{r} e_{n}
$$

Such an expression is adequate when $\frac{d x^{0}}{d s}$ does not change significantly across the wavefront.

However, in the full ray co-ordinate system, there are additional terms dependent on the rates of change of $e_{\text {s }}$ perpendicular to the cenual ray; these terms usually need to be included in order to obtain an adequate description of $\nabla\left(\frac{d \mathbf{x}^{0}}{d s}\right)$. 